\title{
Spin- and valley-dependent magnetotransport in periodically modulated silicene
}

\author{
Kh. Shakouri, ${ }^{1}$ P. Vasilopoulos, ${ }^{2}$ V. Vargiamidis, ${ }^{2}$ and F. M. Peeters ${ }^{1}$ \\ ${ }^{1}$ Departement Fysica, Universiteit Antwerpen Groenenborgerlaan 171, B-2020 Antwerpen, Belgium \\ ${ }^{2}$ Department of Physics, Concordia University, 7141 Sherbrooke Ouest Montréal, Québec, Canada H4B 1R6
}

(Received 16 May 2014; revised manuscript received 3 September 2014; published 25 September 2014)

\begin{abstract}
The low-energy physics of silicene is described by Dirac fermions with a strong spin-orbit interaction and its band structure can be controlled by an external perpendicular electric field $E_{z}$. We investigate the commensurability oscillations in silicene modulated by a weak periodic potential $V=V_{0} \cos \left(2 \pi y / a_{0}\right)$ with $a_{0}$ as its period, in the presence of a perpendicular magnetic field $B$ and of a weak sinusoidal electric field $E_{z}=E_{0} \cos \left(2 \pi y / b_{0}\right)$, where $b_{0}$ is its period. We show that the spin and valley degeneracy of the Landau levels is lifted, due to the modulation, and that the interplay between the strong spin-orbit interaction and the potential and electric field modulations can result in spin- and valley-resolved magnetotransport. At very weak magnetic fields the commensurability oscillations induced by a weak potential modulation can exhibit a beating pattern depending on the strength of the homogenous electric field $E_{z}$ but this is not the case when only $E_{z}$ is modulated. The Hall conductivity plateaus acquire a step structure, due to spin and valley intra-Landau-level transitions, that is absent in unmodulated silicene. The results are critically contrasted with those for graphene and the two-dimensional electron gas.
\end{abstract}

DOI: 10.1103/PhysRevB.90.125444

PACS number(s): 75.47.- m, 85.75.-d

\section{INTRODUCTION}

Silicene, a monolayer of silicon with honeycomb structure, has recently attracted considerable attention [1] and several attempts have been made to synthesize it [2]. Although freestanding silicene has not yet been observed, its stability was predicted [3] and recent theoretical studies predict that it can be stable on nonmetallic surfaces such as graphene [4], boron nitride or $\mathrm{SiC}$ [5], and in graphene-silicene-graphene structures [6]. Moreover, very recently local formation of high-buckled silicene nanosheets has been realized on a $\mathrm{MoS}_{2}$ surface [7].

Silicene is known as the low-buckled counterpart of graphene, but it has gapped Dirac cones. Silicon ( $\mathrm{Si}$ ) and carbon (C) though belong to the same group of the table of elements, but $\mathrm{Si}$ has a larger ionic radius, which promotes a $s p^{3}$ hybridization, whereas the $s p^{2}$ one is energetically more favorable in C-layered materials. Therefore, in a twodimensional (2D) layer of $\mathrm{Si}$ atoms, the bonding is formed by a mixed $s p^{2}-s p^{3}$ hybridization. This leads to the buckling of the structure referred to above, with one sublattice shifted vertically with respect to the other.

Interestingly, contrary to graphene in which the spin-orbit interaction (SOI) is very weak, silicene has a strong SOI. In addition, the low-buckled geometry of silicene together with strong atomic intrinsic SOIs lead to a gap of $1.55 \mathrm{meV}$ [8] between the conduction and valence bands. This gap further distinguishes silicene from other similar 2D materials, e.g., gapped graphene or monolayer $\mathrm{MoS}_{2}$, because its gap is controllable by an external electric field $E_{z}[9,10]$, which is facilitated by its buckled structure.

The strong SOI of silicene, its controllable band gap, and its compatibility with silicon-based electronic technology has led already to various studies such as the spin-Hall effect [8], the valley-polarized anomalous Hall effect $[9,11]$, the capacitance of an electrically tunable silicene device [12], etc.

Since the SOI can lead to spin-resolved transport, pertinent to spintronics and spin-based quantum computing, it is worth studying it in silicene and contrasting the results with those for graphene in which the SOI is very weak. Although other 2D materials, such as $\mathrm{MoS}_{2}$, offer similar possibilities for the integration of spintronics or valleytronics [13], due to its strong spin-valley coupling, the tunability of the spin-split band gap of silicene is attractive. Thus far, several works studied magnetotransport in silicene [10,14]. In Ref. [10] it is shown that the spin and valley resolution of the $n=0$ Landau level (LL) due to electric field gating of silicene can result in a polarized magneto-optical conductivity. Motivated by this, we show that the spin and valley degeneracy of the next LLs can also be lifted by a periodically modulated electric field $E_{z}=E_{0} \cos \left(2 y / b_{0}\right)$. We then explore the influence of SOI on the commensurability or Weiss oscillations [15] in silicene, in the presence of a perpendicular magnetic field $B \| z$ and of a one-dimensional (1D), weak, and periodic potential $V(y)=V_{0} \cos (C y)$, or of field modulation. We also consider the combined action of both modulations.

The present study is similar to those of Refs. [16,17] for the $2 \mathrm{D}$ electron gas (2DEG), and to those for monolayer $[18,19]$ or bilayer [20] graphene in which, contrary to silicene, the SOI is very weak. However, it is richer in results because of the electric field modulation, absent in these studies, and its combination with the potential modulation that leads to spin and valley splittings. A comparison or contrast with these graphene results is desirable, e.g., with the large-amplitude commensurability oscillations reported in Ref. [16]. We make this contrast and highlight further differences due to the strong SOI and an external electric field $E_{z}$. As will be shown, a potential or field $E_{z}$ modulation can create tunable spinand valley-polarized commensurability oscillations when the weak field $B$ is varied. We assess the consequences of such a resolution on magnetotransport properties, such as the appearance of new Hall conductivity plateaus, and consider the case when both modulations are present.

In Sec. II we present the basic expressions for the unmodulated $(V=0)$ and periodically modulated $(V \neq 0$ or $\left.E_{z} \neq 0\right)$ silicene and the linear-response expressions for the 


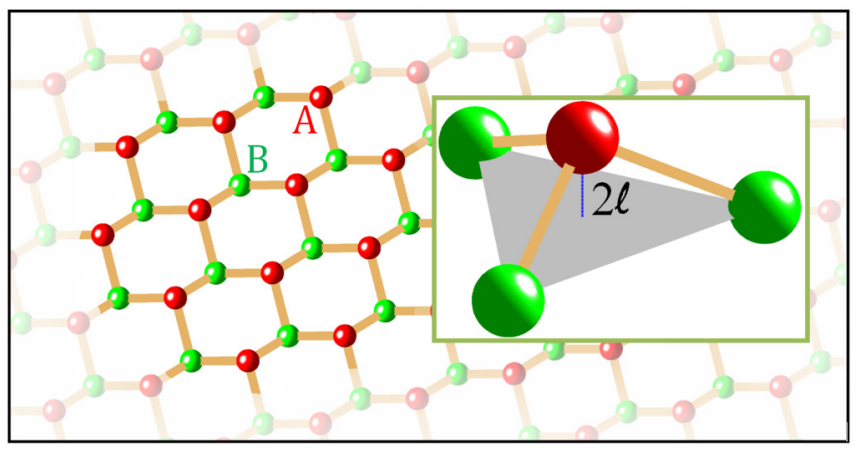

FIG. 1. (Color online) Low-buckled structure of silicene with honeycomb lattice. The vertical distance between the sublattices A and $\mathrm{B}$ is $2 \ell$.

conductivities. The results for the case in which $V(y)$ and $E_{z}(y)$ both vary sinusoidally are shown in Sec. III for the diffusive conductivity and the spin and valley polarizations. The collisional and Hall conductivities and the corresponding resistivities are also given in this section. We conclude with a summary in Sec. IV.

\section{BASIC FORMALISM}

\section{A. Unmodulated silicene}

Silicene is a 2D layer of silicon atoms that forms a lowbuckled honeycomb lattice, see Fig. 1. If we neglect the Rashba SOI, which is very small compared to intrinsic SOI [8], the effective Hamiltonian of low-energy fermions near the $K$ and $K^{\prime}$ valleys is given by $[8,10]$

$$
H=v_{F}\left(\pi_{x} \sigma_{x}-\tau \pi_{y} \sigma_{y}\right)-\left(\tau s_{z} \lambda_{\text {so }}-\ell E_{z}\right) \sigma_{z} .
$$

Here $v_{F}$ is the Fermi velocity, $\tau=+(-)$ is the valley index for the $K\left(K^{\prime}\right)$ valley, and $\vec{\pi}$ is the canonical momentum; $\sigma_{i}$ $(i=x, y, z)$ denotes the pseudospin Pauli matrices, $2 \ell$ is the vertical distance between the two sublattices, and $E_{z}$ is an electric field applied perpendicular to the silicene sheet (here, for brevity, it is assumed that $e E_{z} \rightarrow E_{z}$ ). Furthermore, $\lambda_{\text {so }}$ is the strength of the intrinsic SOI and $s_{z}=1\left(s_{z}=-1\right)$ the up (down) fermion spin. The Hamiltonian matrix $H_{ \pm}$, with the $+(-)$ sign pertaining to the $K\left(K^{\prime}\right)$ valley, takes the form

$$
H_{ \pm}=\left[\begin{array}{cc}
\lambda_{ \pm}\left(s_{z}\right) & v_{F} \pi_{ \pm} \\
v_{F} \pi_{\mp} & -\lambda_{ \pm}\left(s_{z}\right)
\end{array}\right]
$$

with $\lambda_{ \pm}\left(s_{z}\right)=\mp \lambda_{\mathrm{so}} s_{z}+\ell E_{z}$ and $\pi_{ \pm}=\pi_{x} \pm i \pi_{y} ; \pi_{\mu}$ is the $\mu$ component of the shifted momentum operator $\vec{\pi}=p+e \vec{A}$, $\vec{p}$ is the momentum operator, $\vec{A}$ is the vector potential, and $e$ is the electron charge. Now assume that a perpendicular magnetic field $\vec{B}=B \hat{e}_{z}$ is applied to the silicene sheet. We use the Landau gauge $\vec{A}=(-B y, 0,0)$ and write the wave function in the ansatz $\Psi(x, y)=e^{i k_{x} x} \psi(y) / \sqrt{L_{x}}$. This results in the reduction of 2D momentum operators into a $1 \mathrm{D}$ form as $\pi_{ \pm}=\pi_{x} \pm i \pi_{y}=\hbar\left(k_{x} \pm \partial_{y}-y / l_{B}^{2}\right)$, where $l_{B}=\sqrt{\hbar / e B}$ is the magnetic length. In order to achieve a dimensionless form of the Hamiltonian we set $\xi=y / l_{B}-l_{B} k_{x}$; this yields

$$
H_{ \pm}=\hbar \omega_{c}\left[\begin{array}{cc}
\bar{\lambda}_{ \pm}\left(s_{z}\right) & -a_{ \pm} \\
-a_{\mp} & -\bar{\lambda}_{ \pm}\left(s_{z}\right)
\end{array}\right],
$$

where $\bar{\lambda}_{ \pm}=\lambda_{ \pm} / \hbar \omega_{c}\left(\omega_{c}=\sqrt{2} v_{F} / l_{B}\right)$, and $a_{ \pm}=\left(\xi \mp \partial_{\xi}\right) /$ $\sqrt{2}$ are the ladder operators that act on the harmonic oscillator wave functions. The eigenvalues corresponding to Eq. (3) are

$$
E_{n, s_{z}, p}^{ \pm}=p \hbar \omega_{c}\left\{n+\left[\bar{\lambda}_{ \pm}\left(s_{z}\right)\right]^{2}\right\}^{1 / 2} .
$$

Here $p=+1(-1)$ labels the electron (hole) states and $n(n \geqslant 1)$ is the Landau-level (LL) index. Note that the energy is degenerate in the quantum number $k_{x}$. The associated spatial eigenfunctions of an electron near the $K$ valley are

$$
\psi_{n}^{+}(\xi)=\left(\begin{array}{c}
\eta_{1}^{+} \phi_{n}(\xi) \\
\eta_{2}^{+} \phi_{n-1}(\xi)
\end{array}\right),
$$

with $\phi_{n}(\xi)$ as the normalized Harmonic oscillator function and

$$
\begin{aligned}
& \eta_{1}^{+}=\left[\frac{\lambda_{+}\left(s_{z}\right)+E_{n, s_{z}, p}^{+}}{2 E_{n, s_{z}, p}^{+}}\right]^{1 / 2}, \\
& \eta_{2}^{+}=-p\left[\frac{E_{n, s_{z}, p}^{+}-\lambda_{+}\left(s_{z}\right)}{2 E_{n, s_{z}, p}^{+}}\right]^{1 / 2} .
\end{aligned}
$$

For an electron near the $K^{\prime}$ valley the eigenfunctions are

$$
\psi_{n}^{-}(\xi)=\left(\begin{array}{c}
\eta_{1}^{-} \phi_{n-1}(\xi) \\
\eta_{2}^{-} \phi_{n}(\xi)
\end{array}\right),
$$

with

$$
\begin{aligned}
& \eta_{1}^{-}=-p\left[\frac{E_{n, s_{z}, p}^{-}+\lambda_{-}\left(s_{z}\right)}{2 E_{n, s_{z}, p}^{-}}\right]^{1 / 2}, \\
& \eta_{2}^{-}=\left[\frac{E_{n, s_{z}, p}^{-}-\lambda_{-}\left(s_{z}\right)}{2 E_{n, s_{z}, p}^{-}}\right]^{1 / 2} .
\end{aligned}
$$

For $n=0$ each of the $K, K^{\prime}$ valleys involves only one solution of the form $E_{0, s_{z}}^{ \pm}= \pm \hbar \omega_{c} \lambda_{ \pm}\left(s_{z}\right)$ and the associated eigenfunctions are given by $\psi_{0}^{+}=\left[\phi_{0}(\xi), 0\right]^{T}$ and $\psi_{0}^{-}=$ $\left[0, \phi_{0}(\xi)\right]^{T}$, respectively, with $T$ denoting the transpose.

The electron energies at the $K$ and $K^{\prime}$ valleys are related by $E_{n, \pm 1, p}^{+}=E_{n, \mp 1, p}^{-}, n \geqslant 1$, and $E_{0, \pm 1}^{+}=-E_{0, \mp 1}^{-}$.

In Fig. 2 we plot the LLs for the $K$ valley $\left(E_{n, s z, p}^{+}\right)$as a function of the magnetic field $B$. The electric field normal to the silicene sheet is $E_{z}=0$ in Fig. 2(a) and $E_{z}=3.9 \mathrm{meV} / \ell$ in Fig. 2(b). The latter value was chosen to cancel the SOI term for spin up, as in graphene which has a very weak SOI; we refer to it as the "graphene" value. The red dashed (blue solid) curves correspond to the spin-up (spin-down), or $s_{z}=1\left(s_{z}=-1\right)$, states. The insets show the Fermi energy $E_{F}$ versus the field $B$. Every negative (positive) energy curve corresponds to $p=-1$ ( $p=1$ ) except for the lowest LL $n=0$ shown in Fig. 2(b), which is shared by electrons and holes. For $E_{z}=0$ shown in Fig. 2(a), all LLs are twice spin degenerate, cf. Eq. (4), and the $n=0 \mathrm{LL}$ is replaced by two levels, one above and one below the graphene $n=0 \mathrm{LL}$ with zero energy. If we also take the $K^{\prime}$ valley into account, the number of degenerate states corresponding to Landau index $n \neq 0$ is four. In Fig. 2(b) the energy of the lowest LL $(n=0)$ is zero for spin up, 

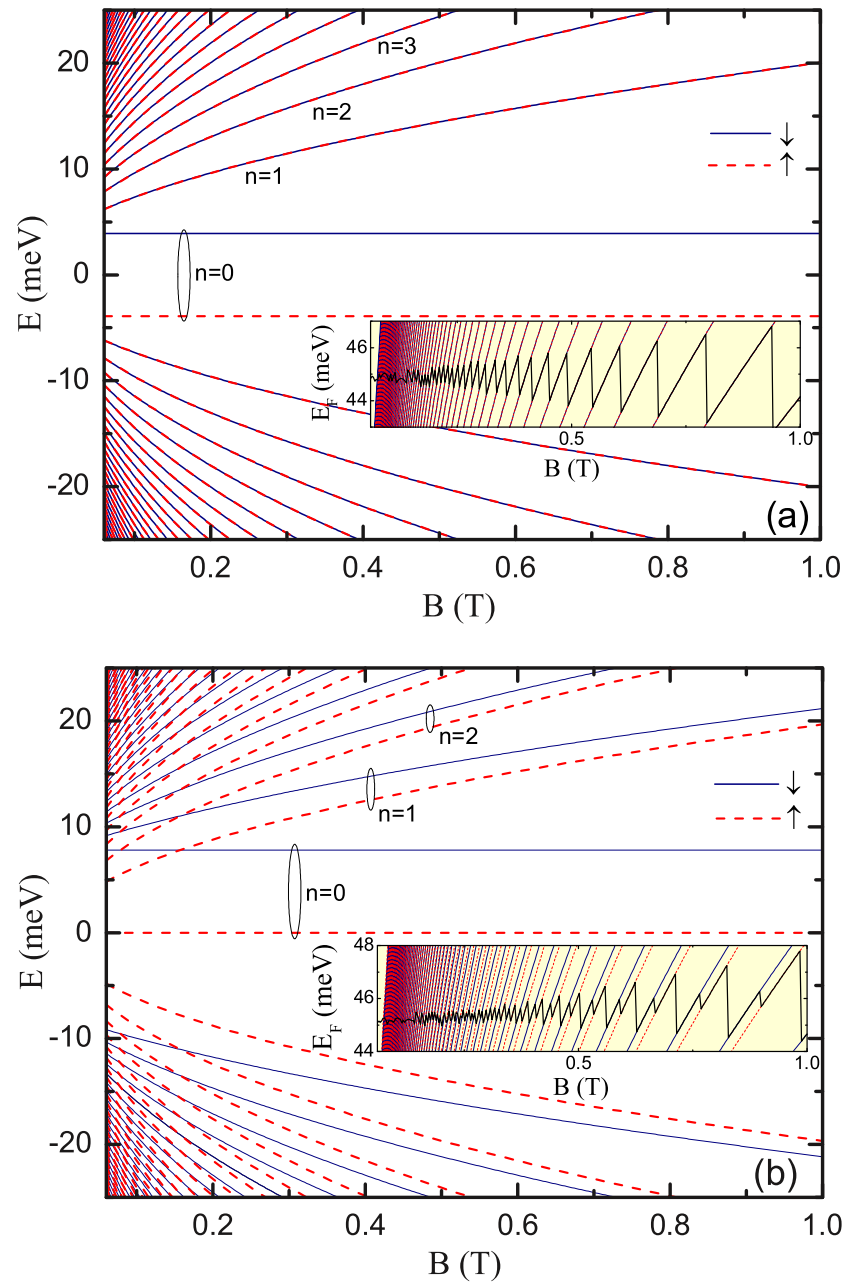

FIG. 2. (Color online) The LLs for the $K$ valley $\left(E_{n, s_{z}, p}^{+}\right)$as a function of the magnetic field. The electric field normal to the silicene sheet is $E_{z}=0$ in (a) and $E_{z}=3.9 \mathrm{meV} / \ell$ in (b). The dashed (solid) curves correspond to the spin-up (spin-down) states and $n$ is the LL index. The strength of the intrinsic SOI is $\lambda_{\text {so }}=3.9 \mathrm{meV}$ and $v_{F}=5.42 \times 10^{5} \mathrm{~m} / \mathrm{s}$ [8]. The insets show $E_{F}$ versus the field $B$ for the electron density $n_{e}=5 \times 10^{11} \mathrm{~cm}^{-2}$ together with the LLs.

like the lowest $\mathrm{LL}$ in a pristine graphene sheet. The reason is that the value $E_{z}=3.9 \mathrm{meV} / \ell$ cancels the SOI term $\lambda_{\text {so }}$ only for spins up. If we reverse the direction of $E_{z}$ then this level will be for spins down. Note that for the $K^{\prime}$ valley the results are the same but the corresponding spin-up and spin-down curves are interchanged. Due to spin splitting in the LLs of Fig. 2(b), small sharp transitions occur in the oscillations of $E_{F}$.

\section{B. Modulated silicene}

In this section we address the effects arising from an external modulation potential and/or that from a modulated field $E_{z}$. As in the case of a two-dimensional electron gas $[15,16]$ or graphene $[18,19]$ the main effect of either modulation is to broaden the LLs into oscillatory energy bands. In addition, we show that when both modulations are present they split the LLs into four branches due to the spin and valley degrees of freedom. This leads to spin- or valley-polarized magnetotransport.

Potential modulation. We consider a weak, one-dimensional, periodic potential $V(y)=V_{0} \cos (C y)$, $C=2 \pi / a_{0}$, with $V_{0}$ and $a_{0}$ as its amplitude and period, respectively. For $V_{0}$ weak we can use first-order perturbation theory to find the energy correction to the eigenvalues (4). We rewrite $V(y)$ in terms of the dimensionless variable $\xi$ as $V(\xi)=V_{0} \cos \left[C l_{B}\left(\xi+l_{B} k_{x}\right)\right]$. Using the unperturbed eigenfunctions of Sec. II A, we find the energy correction for an electron near the $K, K^{\prime}$ valleys as

$$
\begin{aligned}
\Delta E_{n, s_{z}, p}^{ \pm}\left(k_{x}\right) & =\left\langle n, s_{z}, p, k_{x}|V(\xi)| n, s_{z}, p, k_{x}\right\rangle \\
& =V_{0} \cos \left(C x_{0}\right) e^{-u / 2} G_{n, s_{z}, p}^{ \pm} ;
\end{aligned}
$$

here $x_{0}=l_{B}^{2} k_{x}, u=C^{2} l_{B}^{2} / 2$,

$$
\begin{aligned}
& G_{n, s_{z}, p}^{+}=\left|\eta_{1}^{+}\right|^{2} L_{n}(u)+\left|\eta_{2}^{+}\right|^{2} L_{n-1}(u), \\
& G_{n, s_{z}, p}^{-}=\left|\eta_{1}^{-}\right|^{2} L_{n-1}(u)+\left|\eta_{2}^{-}\right|^{2} L_{n}(u),
\end{aligned}
$$

and $L_{n}(u)$ are the Laguerre polynomials. The energy correction depends on the wave vector $k_{x}$ because the potential along the $y$ direction breaks the $x, y$ spatial symmetry of the unmodulated case. That is, the periodic potential broadens the LLs, with constant energy, into bands. Given the oscillatory nature of the Laguerre polynomials for large $n$, in addition to cosine function $\cos \left(C x_{0}\right)$, one easily sees that the bandwidths (9) oscillate with the magnetic field $B$. For $n=0$ the energy correction is

$$
\Delta E_{0, s_{z}}^{ \pm}\left(k_{x}\right)=V_{0} \cos \left(C x_{0}\right) e^{-u / 2} .
$$

Note that the bandwidth variations (9) are distinct for spins up and down because the coefficients $\eta_{1,2}^{ \pm}$in Eq. (10) are spin dependent.

Field modulation. In the presence of a sinusoidal modulated field $E_{z}(y)=E_{0} \cos (D y), D=2 \pi / b_{0}$, with $E_{0}$ and $b_{0}$ as its amplitude and period, respectively, the first-order energy corrections for an electron near the $K, K^{\prime}$ valleys are given by

$$
\begin{aligned}
\Delta^{\prime} E_{n, s_{z}, p}^{ \pm}\left(k_{x}\right) & =\left\langle n, s_{z}, p, k_{x}\left|\ell E_{z}(\xi) \sigma_{z}\right| n, s_{z}, p, k_{x}\right\rangle \\
& =\ell E_{0} \cos \left(D x_{0}\right) e^{-u^{\prime} / 2} G_{n, s_{z}, p}^{\prime \pm},
\end{aligned}
$$

where $u^{\prime}=D^{2} l_{B}^{2} / 2$ and

$$
\begin{aligned}
& G_{n, s_{z}, p}^{\prime+}=\left|\eta_{1}^{+}\right|^{2} L_{n}\left(u^{\prime}\right)-\left|\eta_{2}^{+}\right|^{2} L_{n-1}\left(u^{\prime}\right), \\
& G_{n, s_{z}, p}^{\prime-}=\left|\eta_{1}^{-}\right|^{2} L_{n-1}\left(u^{\prime}\right)-\left|\eta_{2}^{-}\right|^{2} L_{n}\left(u^{\prime}\right) .
\end{aligned}
$$

For $n=0$ we find

$$
\Delta^{\prime} E_{0, s_{z}}^{ \pm}\left(k_{x}\right)= \pm \ell E_{0} \cos \left(D x_{0}\right) e^{-u^{\prime} / 2} .
$$

In contrast to the case of potential modulation, the energy correction (14) for the $n=0 \mathrm{LL}$ is different at the $K$ and $K^{\prime}$ valleys.

Density of states. The density of states (DOS), as a function of the energy $E$, is given by $D(E)=\sum_{\zeta} \delta\left(E-E_{\zeta}\right)$. To calculate the DOS we approximate the Dirac $\delta$ function by a Gaussian, i.e., $\delta\left[E-E_{n, s_{z}, p}^{ \pm}\left(k_{x}\right)\right] \approx(1 / \Gamma \sqrt{\pi}) \exp \{-[E-$ $\left.\left.E_{n, s_{z}, p}^{ \pm}\left(k_{x}\right)\right]^{2} / \Gamma^{2}\right\}$, with $\Gamma$ as the broadening parameter. In the 


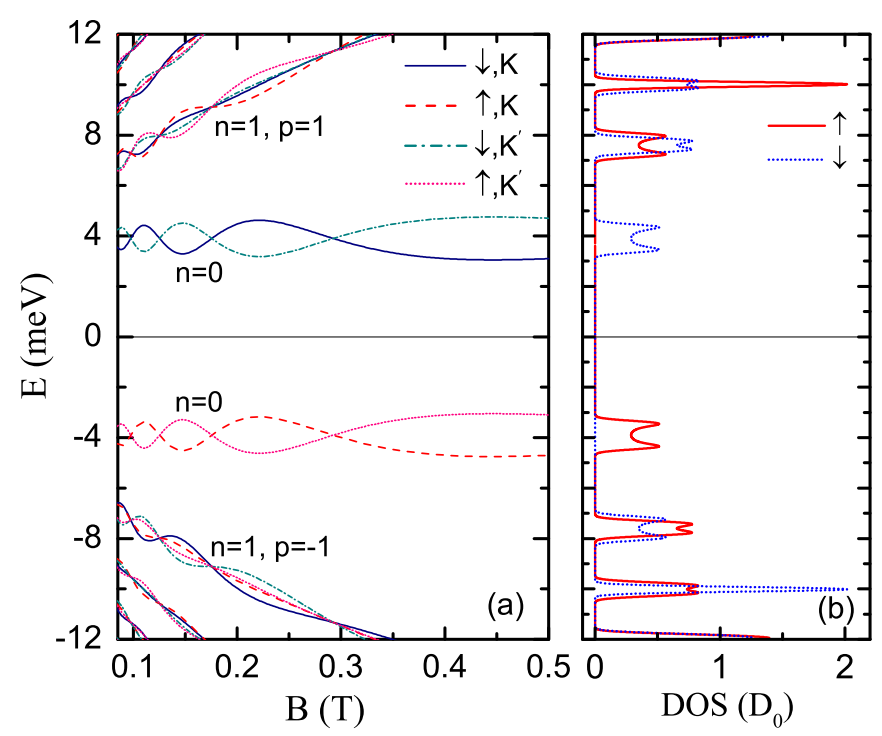

FIG. 3. (Color online) (a) Landau fan diagram for electron-hole states in the presence of a sinusoidal field with period $b_{0}=300 \mathrm{~nm}$ and amplitude $E_{0}=1 \mathrm{meV} / \ell$. The wave vector parallel to the modulation is $k_{x}=10^{8} \mathrm{~m}^{-1}$. As shown, the modulated field $E_{z}$ lift the spin and valley degeneracy of the LLs. Every LL with $n \geqslant 1$ is split into four branches, while the $n=0 \mathrm{LL}$ splits in two. (b) The DOS for up (solid curve) and down (dotted curve) spin states, in units of $D_{0}=\left(2 \pi^{3 / 2} l_{B}^{2} \Gamma\right)^{-1}$, for $B=0.11 \mathrm{~T}$ and $\Gamma=0.02 \hbar \omega_{c}$. Notice that the peaks corresponding to the $n=0 \mathrm{LL}$ are fully spin polarized.

presence of a weak periodic modulation this yields

$\frac{D(E)}{S_{0} D_{0}}=\frac{1}{2 \pi} \int_{0}^{2 \pi} d \theta \sum_{n, s_{z}, p, \pm} e^{-\left[E-E_{n, s_{z}, p}^{ \pm}-\Delta E_{n, s_{z}, p}^{ \pm}(\theta)\right]^{2} / \Gamma^{2}}$,

where $D_{0}=\left(2 \pi^{3 / 2} l_{B}^{2} \Gamma\right)^{-1}, \theta=C x_{0}$ (for potential modulation), and $S_{0}=a_{0} L_{x}$.

In Figs. 3(a) and 4(a) we show the broadened LLs versus the magnetic field $B$, for $k_{x}=10^{8} \mathrm{~m}^{-1}$, respectively in the presence of a sinusoidal field modulation, with period $b_{0}=$ $300 \mathrm{~nm}$ and strength $E_{0}=1 \mathrm{meV} / \ell$, and of a sinusoidal potential modulation with period $a_{0}=300 \mathrm{~nm}$ and amplitude $V_{0}=1 \mathrm{meV}$. The DOS related to each case is separately shown for the up and down spin states in Figs. 3(b) and 4(b). As shown, the oscillatory $E_{z}$ lifts the spin and valley degeneracy of the LLs, however for $V_{0}$ modulation and $E_{z}=0$ the LLs are still valley degenerate. For modulated $E_{z}$, shown in Fig. 3(a), every LL is split into four branches except for certain values of $B$ for which $\cos \left(D x_{0}\right)=0$ and the bandwidth vanishes. That occurs at fields $B=B_{c} /(2 m+1)$, with $B_{c}=2(h / e) k_{x} / \pi b_{0}$ and $m$ is a nonnegative integer. Notice that the $n=0 \mathrm{LL}$ splits in two valley branches that have the same spin as can be seen from the eigenvalue $E_{0, s_{z}}^{ \pm}=-\hbar \omega_{c} \lambda_{\mathrm{so}} s_{z}+\Delta^{\prime} E_{0, s_{z}}^{ \pm}$. For positive (negative) energies this spin is the down (up); also see the DOS for up and down spins shown in Figs. 3(b) and 4(b).

The lifting of the spin and valley degeneracy results from the fact that for $\ell E_{0}=0$ the unperturbed eigenvalues (4) are spin and valley degenerate, $E_{n, s_{z}= \pm 1, p}^{+}=E_{n, s_{z}= \pm 1, p}^{-}=$ $p \hbar \omega_{c}\left(n+\bar{\lambda}_{\text {so }}^{2}\right)^{1 / 2}$, whereas the coefficients $\eta_{1,2}^{ \pm}$depend on the spin and valley: we have, e.g., $\left.\left(\eta_{1}^{+}\right)^{2}\right|_{s_{z}= \pm 1}=\left.\left(\eta_{2}^{-}\right)^{2}\right|_{s_{z}=\mp 1}=$ $\left[\left(\mp \lambda_{\text {so }}+E_{n, s_{z}, p}^{+}\right) / 2 E_{n, s_{z}, p}^{+}\right]$, etc. Accordingly, all energy cor-

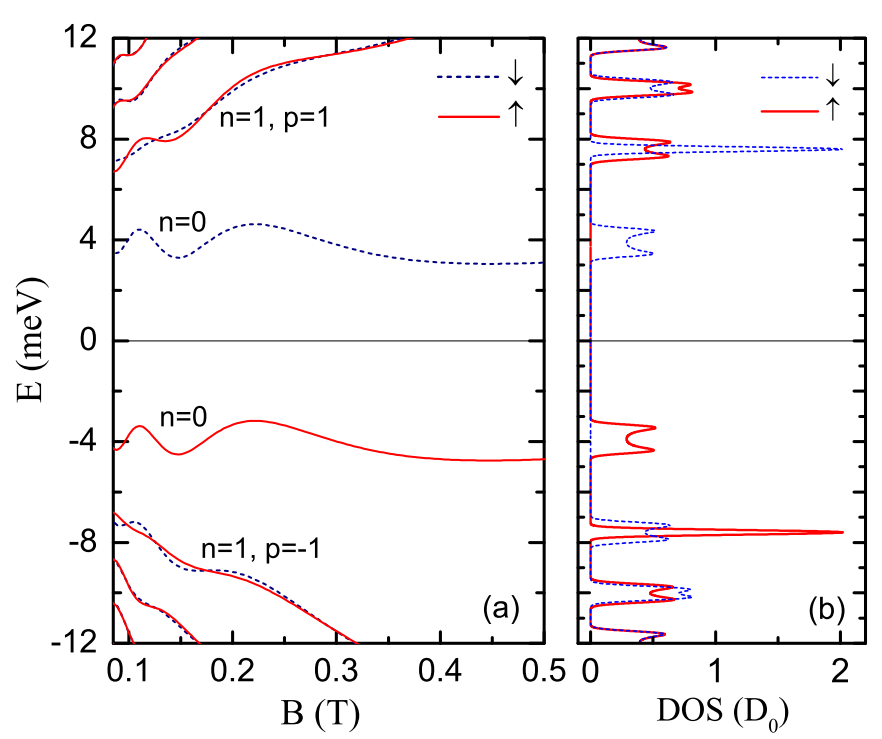

FIG. 4. (Color online) (a) Landau fan diagram for electron-hole states in the presence of a single potential modulation with period $a_{0}=300 \mathrm{~nm}$ and amplitude $V_{0}=1 \mathrm{meV} / \ell$. The wave vector parallel to the modulation is $k_{x}=10^{8} \mathrm{~m}^{-1}$. The LLs are valley degenerate. (b) The DOS for up (solid curve) and down (dashed curve) spin states, in units of $D_{0}=\left(2 \pi^{3 / 2} l_{B}^{2} \Gamma\right)^{-1}$, for $B=0.11 \mathrm{~T}$ and $\Gamma=0.02 \hbar \omega_{c}$.

rections evaluated with Eq. (12) are neither spin nor valley degenerate anymore. Notice that compared to the spin or valley splitting induced by the field modulation, the LL splitting due to the potential modulation is very small. The reason is that for $\bar{\lambda}_{\text {so }} \ll n$ the $\eta_{1,2}^{ \pm}$coefficients are approximately equal and then $G_{n, s_{z}, p}^{+} \approx G_{n, s_{z}, p}^{-}$, while $G_{n, s_{z}, p}^{\prime+}=-G_{n, s_{z}, p}^{\prime-}$. This means that for a potential modulation the LL broadening for the two valleys is the same, whereas for the field modulation the band broadening at the $K, K^{\prime}$ valleys is opposite. The amplitude of the bandwidth oscillations decreases for higher LLs because the modulations are very weak and perturb mostly the lowest LLs.

At very low fields $B$ the function $\cos \left(C x_{0}\right)$ in Eq. (9) fluctuates rapidly but the function $e^{-u / 2}$ decreases the oscillation amplitude and causes the bandwidth oscillations to disappear. The same holds for the function $\cos \left(D x_{0}\right)$. This explains the form of the $n=0 \mathrm{LL}$. Moreover, the argument of $D x_{0}$ decreases for very large fields $B$. For $B$ larger than a critical value $B_{c}$, we have $D x_{0}<\pi / 2$ and the cosine does not oscillate. That is, for $B>B_{c}$, the energies increase monotonically (or decrease for hole states) and the bandwidth ceases to oscillate.

To assess more the effect of broadening on the DOS we plot in Fig. 5 the DOS calculated per area $S_{0}$ for different parameters shown in the caption. One sees the usual van Hove peaks but their heights are finite due to the width $\Gamma \neq 0$ especially when it is large. For fixed electric field $E_{z}=3.9 \mathrm{meV} / l_{z}$, pertaining to the graphenelike case, there is a zero-energy LL available for spins up in the $K$ valley and similarly for spins down in the $K^{\prime}$ valley. Therefore, there is a finite DOS at zero energy available for both electrons and holes. For $E_{z}=0$, with spectrum shown in Fig. 4(a), the LLs are valley degenerate and so the DOS oscillations are sharper and stronger. Note that one obtains the same DOS 

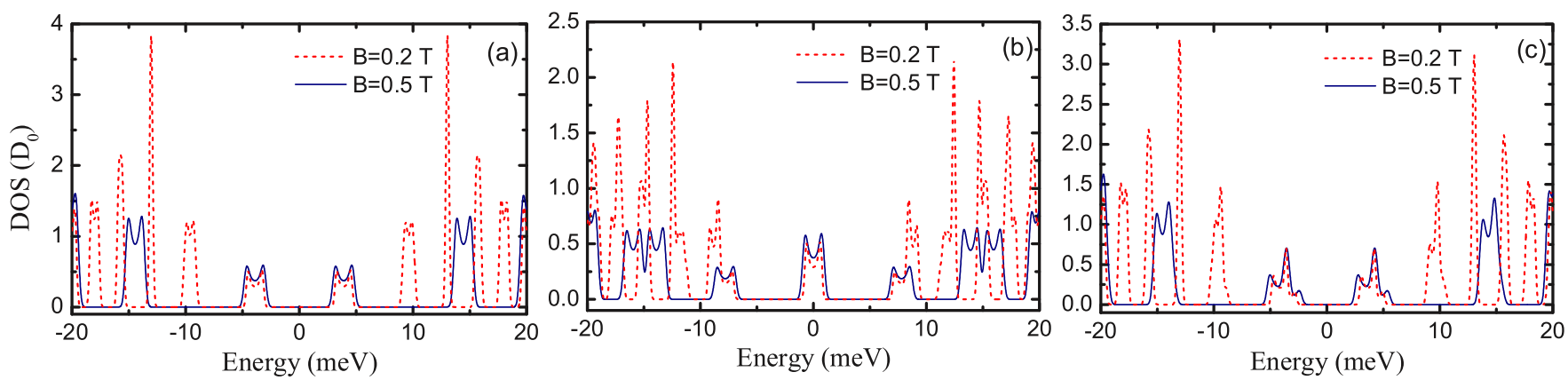

FIG. 5. (Color online) DOS per area $S_{0}=a_{0} L_{x}$, in units of $D_{0}=\left(2 \pi^{3 / 2} l_{B}^{2} \Gamma\right)^{-1}$, as a function of the energy $E$ in the presence of a periodic modulation of amplitude $V_{0}=1 \mathrm{meV}$ and period $a_{0}=300 \mathrm{~nm}$. The results are shown for electric fields (a) $E_{z}=0$ and (b) $E_{z}=3.9 \mathrm{meV} / \ell$. In (c) a sinusoidal field, with amplitude $E_{0}=1 \mathrm{meV} / \ell$ and period $b_{0}=200 \mathrm{~nm}$, is present. The broadening parameter is $\Gamma=0.02 \hbar \omega_{c}$.

by approximating the Dirac $\delta$ function by a Lorentzian of width $\Gamma$.

\section{Linear-response conductivity expressions}

To evaluate the transport coefficients we adopt the formalism of Ref. [21]. In this approach one considers a many-body system described by the Hamiltonian $H=H_{0}+$ $H_{I}-\mathbf{R} \cdot \mathbf{F}(t)$, where $H_{0}$ is the unperturbed part, $H_{I}$ is a binary-type interaction (e.g., between electrons and impurities or phonons), and $-\mathbf{R} \cdot \mathbf{F}(t)$ is the interaction of the system with the external field $F(t)$. For conductivity problems we have $\mathbf{F}(t)=e \mathbf{E}(t)$, where $\mathbf{E}(t)$ is the electric field, $e$ is the electron charge, $\mathbf{R}=\sum_{\mathbf{r}_{i}}$, and $\mathbf{r}_{i}$ is the position operator $\mathbf{r}_{i}$ of electron $i$. In the representation in which $H_{0}$ is diagonal the many-body density operator $\rho=\rho^{d}+\rho^{\text {nd }}$ has a diagonal part $\rho^{d}$ and a nondiagonal part $\rho^{\text {nd }}$. For weak electric fields and weak scattering potentials, for which the first Born approximation applies, the conductivity tensor has a diagonal part $\sigma_{\mu \nu}^{d}$ and a nondiagonal part $\sigma_{\mu \nu}^{\text {nd }}$ with total $\sigma_{\mu \nu}=\sigma_{\mu \nu}^{d}+\sigma_{\mu \nu}^{\text {nd }}$, $\mu, v=x, y$.

In general we have two kinds of currents, diffusive and hopping, but usually only one of them is dominant. In the unmodulated case, for instance, of Sec. II A, $\left\langle\zeta\left|v_{x}\right| \zeta\right\rangle=$ $\left\langle\zeta\left|v_{y}\right| \zeta\right\rangle=0$ and the diffusive current is absent. In the modulated case though, as shown by Eqs. (16) and (17), $\left\langle\zeta\left|v_{x}\right| \zeta\right\rangle \neq 0$ and this results in a diffusive current contribution. The general dc expression for the diffusive conductivity $\sigma_{\mu \nu}^{\mathrm{dif}}$, assuming quasielastic scattering, is given by

$$
\sigma_{\mu \nu}^{\mathrm{dif}}=\frac{\beta e^{2}}{S_{0}} \sum_{\zeta} \tau_{\zeta} f_{\zeta}\left(1-f_{\zeta}\right) v_{\nu \zeta} v_{\mu \zeta}
$$

where $\tau_{\zeta}$ is the momentum relaxation time and $v_{\mu \zeta}$ are the diagonal matrix elements of the velocity operator. Furthermore, $f_{\zeta}=\left[1+\exp \beta\left(E_{\zeta}-E_{F}\right)\right]^{-1}$ is the Fermi-Dirac function with $\beta=1 / k_{B} T$ and $T$ is the temperature.

In the presence of a magnetic field, with or without modulation, there exists a collisional or hopping contribution. It is given by [21]

$$
\sigma_{\mu \mu}^{\mathrm{col}}=\frac{\beta e^{2}}{2 S_{0}} \sum_{\zeta, \zeta^{\prime}} W_{\zeta, \zeta^{\prime}} f\left(E_{\zeta}\right)\left[1-f\left(E_{\zeta^{\prime}}\right)\right]\left(\alpha_{\mu}^{\zeta}-\alpha_{\mu}^{\zeta^{\prime}}\right)^{2}
$$

Here $W_{\zeta, \zeta^{\prime}}$ is the scattering rate between the perturbed states $|\zeta\rangle$ and $\left|\zeta^{\prime}\right\rangle$, and $\alpha_{\mu}^{\zeta}$ denotes the expectation value of the position operator $\alpha_{\mu}$. $W_{\zeta, \zeta^{\prime}}$ will be specified later in Sec. III D.

Regarding the contribution $\sigma_{\mu \nu}^{\text {nd }}$ one can use [22] the identity $f_{\zeta}\left(1-f_{\zeta^{\prime}}\right)\left[1-\exp \beta\left(E_{\zeta}-E_{\zeta^{\prime}}\right)\right]=f_{\zeta}-f_{\zeta^{\prime}}$ and cast the original form [21] in the more familiar one

$$
\sigma_{\mu \nu}^{\mathrm{nd}}=\frac{i \hbar e^{2}}{S_{0}} \sum_{\zeta \neq \zeta^{\prime}} \frac{\left(f_{\zeta}-f_{\zeta^{\prime}}\right) v_{\nu \zeta \zeta^{\prime}} v_{\mu \zeta^{\prime} \zeta}}{\left(E_{\zeta}-E_{\zeta^{\prime}}\right)\left(E_{\zeta}-E_{\zeta^{\prime}}+i \Gamma_{\zeta}\right)},
$$

where $v_{\nu \zeta \zeta^{\prime}}$ and $v_{\mu \zeta \zeta^{\prime}}$ are the nondiagonal matrix elements of the velocity operator and $\mu, v=x, y$. The sum runs over all quantum numbers $|\zeta\rangle=\left|n, k_{x}, s_{z}, p, \tau\right\rangle$ and $\left|\zeta^{\prime}\right\rangle=$ $\left|n^{\prime}, k_{x}^{\prime}, s_{z}^{\prime}, p^{\prime}, \tau^{\prime}\right\rangle$ provided $\zeta \neq \zeta^{\prime}$. The infinitesimal quantity $\epsilon$ in the original form [21] has been replaced by $\Gamma_{\zeta}$ to account for the broadening of the energy levels.

In the following we will evaluate expressions (16)-(18) for electron scattering by diluted impurities.

\section{RESULTS}

\section{A. Weak 1D periodic modulation}

In the presence of a weak periodic modulation the $x, y$ spatial symmetry of unmodulated silicene is perturbed, giving rise to a broadening of the LLs into bands, cf. Eqs. (9)(14). This broadening induces a group velocity proportional to the corresponding bandwidth of each LL, that results in a diffusive conductivity. The electron velocity in the $n$th Landau band induced by the modulation is given by $\left[v_{x, n, s_{z}, p}^{ \pm}\left(k_{x}\right)=\left(\partial / \partial k_{x}\right) \Delta E_{n, s_{z}, p}^{ \pm} / \hbar\right]$

$$
v_{x, n, s_{z}, p}^{ \pm}\left(k_{x}\right)=-\frac{V_{0} C l_{B}^{2}}{\hbar} \sin \left(C x_{0}\right) e^{-u / 2} G_{n, s_{z}, p}^{ \pm}(u),
$$

and that by the field modulation

$$
v_{x, n, s_{z}, p}^{\prime \pm}\left(k_{x}\right)=-\frac{\ell E_{0} D l_{B}^{2}}{\hbar} \sin \left(D x_{0}\right) e^{-u^{\prime} / 2} G_{n, s_{z}, p}^{\prime \pm}\left(u^{\prime}\right) .
$$

When temperature is sufficiently low, the relaxation time is approximately constant and equal to its value at the Fermi energy $\tau_{\zeta} \approx \tau_{F}$. In addition, $\beta f\left(E_{\zeta}\right)\left[1-f\left(E_{\zeta}\right)\right]=-\partial f /\left.\partial E\right|_{E=E_{\zeta}}$. Since the modulation is very weak, the variation of $k_{x}$ does not affect much the Fermi distribution at low magnetic fields. The magnetic field-induced flux density [23] $B / \phi_{0}=1 / 2 \pi l_{B}^{2}$ ( $\phi_{0}=h / e$ is the flux quantum) decreases linearly for low fields $B$ and the resulting capacity of each LL for filling with 


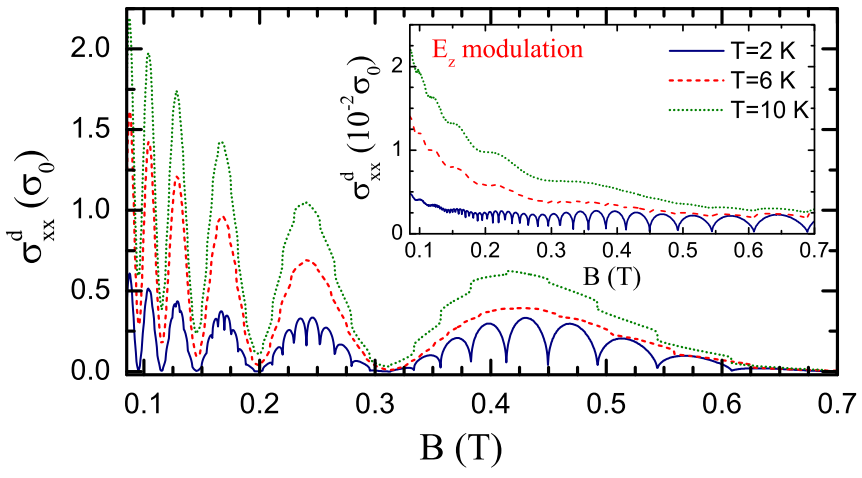

FIG. 6. (Color online) Diffusive conductivity induced by only weak $V(y)$ modulation, in units of $\sigma_{0}=\left(e^{2} / h\right)\left(\beta V_{0}^{2} \tau / h\right)$, versus the magnetic field $B$ for different temperatures. The contributions of both spin states and valleys are included. The parameters used are $V_{0}=$ $1 \mathrm{meV}, a_{0}=300 \mathrm{~nm}, n_{e}=5 \times 10^{11} \mathrm{~cm}^{-2}$, and $v_{F}=5.42 \times 10^{5} \mathrm{~m} / \mathrm{s}$. The inset shows the same conductivity but in the presence of field modulation with strength $E_{0}=1 \mathrm{meV} / \ell$ and amplitude $b_{0}=$ $300 \mathrm{~nm}$. Notice that the oscillation amplitude is much smaller in this case.

electrons diminishes. Therefore, the LL index pertinent to the Fermi energy $n_{F}$ is larger for lower fields $B$ and, as discussed in Sec II B, the bandwidth is smaller for higher LLs. Hence, one can remove the $k_{x}$ dependence from the Fermi functions. In this case, using the prescription $\left(L_{y} / l_{B}^{2}=k_{0}\right)$

$$
\sum_{\zeta} \rightarrow \frac{L_{x}}{2 \pi} \int_{0}^{k_{0}} d k_{x} \sum_{n, s_{z}, p, \pm}
$$

and from Eqs. (16) and (19) we obtain the diffusive conductivity due to the potential modulation as

$$
\sigma_{x x}^{\mathrm{dif}}=\frac{e^{2}}{h} \frac{\beta V_{0}^{2} \tau}{\hbar} u e^{-u} \sum_{n, s_{z}, p, \pm} f_{\zeta}\left(1-f_{\zeta}\right)\left(G_{n, s_{z}, p}^{ \pm}\right)^{2},
$$

where $f_{\zeta}=f\left(E_{\zeta}\right)=f\left(E_{n, s_{z}, p, \pm}\right)$. For a field modulation we obtain Eq. (21) with $V_{0}, u$, and $G$ replaced by $\ell E_{0}, u^{\prime}$, and $G^{\prime}$, respectively. When $n$ is large we can use the approximation $e^{-u / 2} L_{n}(u) \approx\left(\pi^{2} n u\right)^{-1 / 4} \cos (2 \sqrt{n u}-\pi / 4)+O\left(n^{-3 / 4}\right)$; and the zeros of $e^{-u / 2} L_{n}(u)$ are given by $u=[\pi / 2(m+3 / 4)]^{2} / n$.

The longitudinal diffusive conductivity $\sigma_{x x}^{d}$ induced by only a sinusoidal potential modulation with amplitude $V_{0}=$ $1 \mathrm{meV}$ and period $a_{0}=300 \mathrm{~nm}$ is shown in Fig. 6. The inset displays the results for a $E_{z}$ modulation with strength $E_{0}=1 \mathrm{meV} / \ell$ and period $b_{0}=300 \mathrm{~nm}$. In both cases, the oscillation amplitude increases with temperature because the main contribution to the diffusive conductivity comes from the LLs near the Fermi level $E_{F}$ with an energy spacing smaller than a few $k_{B} T$. Indeed, an increase in the temperature broadens the function $f\left(E_{\zeta}\right)\left[1-f\left(E_{\zeta}\right)\right]$ and allows more LLs to contribute to the diffusive conductivity. This results in an increase of the oscillation amplitude. However, for higher magnetic fields the oscillations are damped again. This is due to the increasing LL spacing by the magnetic field so that the number of LLs captured by the thermal broadening $k_{B} T$ decreases (e.g., note that at $T=2 \mathrm{~K}$ the thermal broadening is about $0.17 \mathrm{meV}$, whereas the LL spacing at high $B$ exceeds several $\mathrm{meV}$ ). A comparison between the

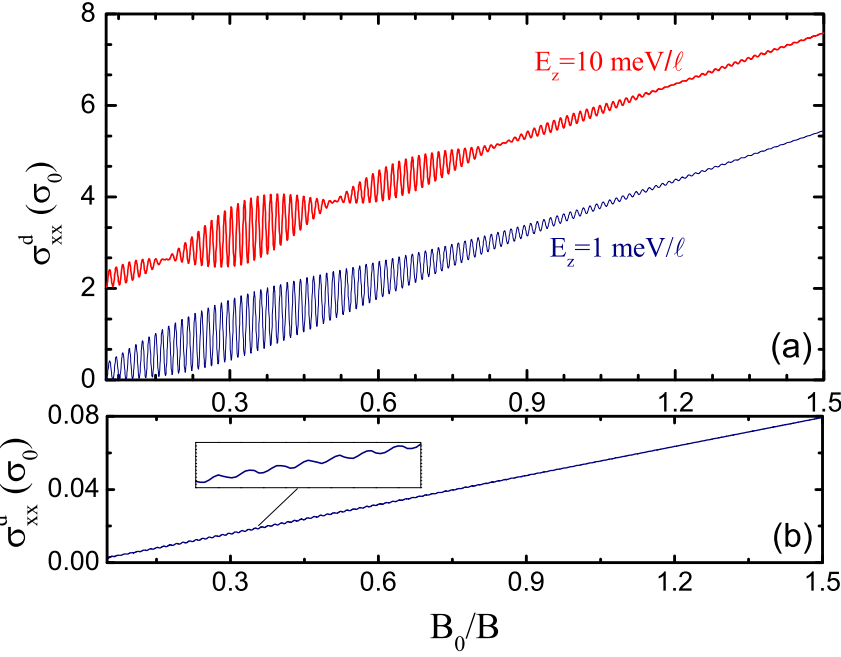

FIG. 7. (Color online) (a) Diffusive conductivity versus inverse magnetic field for a potential modulation with $V_{0}=1 \mathrm{meV}$ and $a_{0}=$ $300 \mathrm{~nm}$. The curves are for constant electric fields and the one for $E_{z}=10 \mathrm{meV} / \ell$ is shifted up by $2 \sigma_{0}$ for clarity. The beating pattern is best seen only for $E_{z}=10 \mathrm{meV} / \ell$. (b) The same as in (a) for an electric field modulation with $E_{0}=1 \mathrm{meV} / \ell$ and $b_{0}=300 \mathrm{~nm}$. The electron density is $n_{e}=5 \times 10^{11} \mathrm{~cm}^{-2}$ and $T=2 \mathrm{~K}$.

results of the $V(y)$ and $E_{z}$ modulations demonstrates that the amplitude of the Weiss oscillations is much larger for a potential modulation which is a reasonable consequence of the fact that $\left|G_{n, s_{z}, p}^{ \pm}\right| \gg\left|G_{n, s_{z}, p}^{\prime \pm}\right|$.

When the magnetic field is very weak and the number of filled LLs is large, an asymptotic analytic expression can be derived for the commensurability oscillations of the conductivity similar to the case of 2DEG [16]. As shown in Fig. 7(a) and in Ref. [14], the commensurability oscillations induced by a weak potential modulation can exhibit a regular beating pattern versus the inverse magnetic field $B_{0} / B, B_{0}$ being the field where the magnetic length $l_{B}$ is equal to the period $a_{0}$. Nevertheless, this occurs for a relatively strong electric field which has to be even stronger when $a_{0}$ is decreased. In contrast, with only the electric field $E_{z}$ modulation present, not treated in Ref. [14], no beating pattern is seen in these oscillations and their amplitude decreases monotonically with $B_{0} / B$.

The interplay between the SOIs and external modulations can result in a spin polarization. To show this, we plot in Fig. 8 the spin polarization obtained for the diffusive conductivity, i.e., $p_{s}=\left(\sigma_{x x, \uparrow}^{\mathrm{dif}}-\sigma_{x x, \downarrow}^{\mathrm{dif}}\right) / \sigma_{x x}^{\mathrm{dif}}$, as a function of the magnetic field $B$. For the case of field modulation, $p_{s}$ exhibits relatively regular oscillations but the period increases with $B$ because of the enhancement in the period of the bandwidth oscillations, cf. Sec. II B. This implies that for field modulation the Weiss oscillations are spin resolved so that a nearly perfect spin polarization is achievable in certain ranges of $B$ values. Note that the valley polarization $p_{v}=\left(\sigma_{x x,+}^{\mathrm{dif}}-\sigma_{x x,-}^{\mathrm{dif}}\right) / \sigma_{x x}^{\mathrm{dif}}$ vanishes because $v_{x}^{\prime+}=-v_{x}^{\prime-}$, i.e., the electrons from the $K$ and $K^{\prime}$ valleys contribute equally.

With only the $V_{0}$ modulation present neither a sizable spin nor valley gap is created in the conductivity oscillations. The $p_{s}$ oscillations appear as local discontinuities whose size is small for the shown $B$ range with the exception of a rather large one near $B \approx 0.43 \mathrm{~T}$. In addition, the valley polarization vanishes 


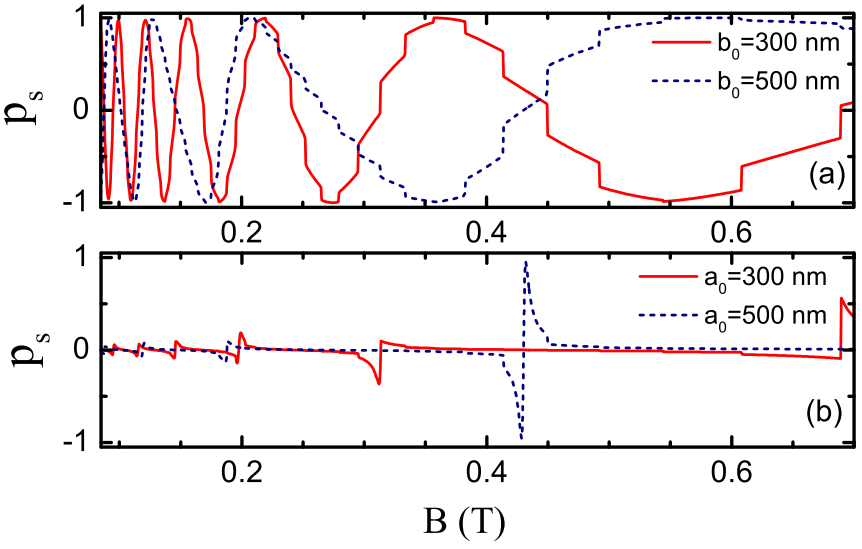

FIG. 8. (Color online) Spin polarization $p_{s}$ versus the magnetic filed $B$ for a single modulation with $E_{0}=1 \mathrm{meV} / \ell$ in (a) and $V_{0}=1 \mathrm{meV}$ in (b) for two different modulation periods. The electron density is $n_{e}=5 \times 10^{11} \mathrm{~cm}^{-2}$ and $T=2$. For specific fields $B$ the spin resolution due to field modulation is nearly perfect and this could be useful for spintronics.

when $E_{z}=0$ since the energy spectrum is valley degenerate and then $v_{x}^{+}=v_{x}^{-}$. Even by applying a large uniform electric field the $K$ and $K^{\prime}$ valleys contribute approximately equally to the diffusive conductivity. The reason is that for large $n$ the spin-dependent coefficients are $\left(\eta_{1,2}^{ \pm}\right)^{2} \approx 1 / 2$ and the functions $G_{n, s z, p}^{ \pm}$pertinent to the different valleys are equal. This shows that to achieve a valley-polarized diffusive current the application of a single modulation is not very helpful.

\section{B. Two weak 1D periodic modulations}

As discussed above, a weak $V_{0}$ modulation cannot create a sizable valley splitting in the Weiss oscillations of the conductivity. On the other hand, by modulating only the electric field the oscillation amplitude is small and so is the valley splitting. Here we demonstrate that a certain combination of field and potential modulations restores the drawbacks when only a single modulation is present: The field and potential modulations lead to a spin- and valley-resolved diffusive current and simultaneously the $V_{0}$ modulation compensates for the reduction in oscillation amplitude when only the field modulation is present. In order to demonstrate this we proceed as follows.

We first assume that a field modulation with strength $E_{0}=$ $1 \mathrm{meV} / \ell$ and period $b_{0}=300 \mathrm{~nm}$ is present. We then calculate the spin $\left(p_{s}\right)$ and valley $\left(p_{v}\right)$ polarization by tuning the strength of the $V(y)$ modulation. The results are summarized in Fig. 9 for temperature $T=2 \mathrm{~K}$, electron density $n_{e}=5 \times 10^{11} \mathrm{~cm}^{-2}$, and fields $B=0.5 \mathrm{~T}$ in Fig. 9(a) and $0.8 \mathrm{~T}$ in Fig. 9(b). As seen for low values of $V_{0} / \ell E_{0}$ with period $a_{0}=b_{0}, p_{v}$ is maximal (for $B=0.8 \mathrm{~T}$ a nearly $100 \%$ valley polarization is reachable). In contrast, $p_{s}$ is maximal for certain periods $a_{0} \neq$ $b_{0}$. In the presence of both modulations the group velocities at the two valleys are no longer equal, that is $\left|v_{x}^{\prime+}+v_{x}^{+}\right| \neq$ $\left|v_{x}^{\prime-}+v_{x}^{-}\right|$, resulting in the valley-resolved Weiss oscillations. By increasing the strength $V_{0}$ the polarization $p_{v}$ starts to fade since the bandwidth induced by the potential modulation strongly dominates that for the field modulation.
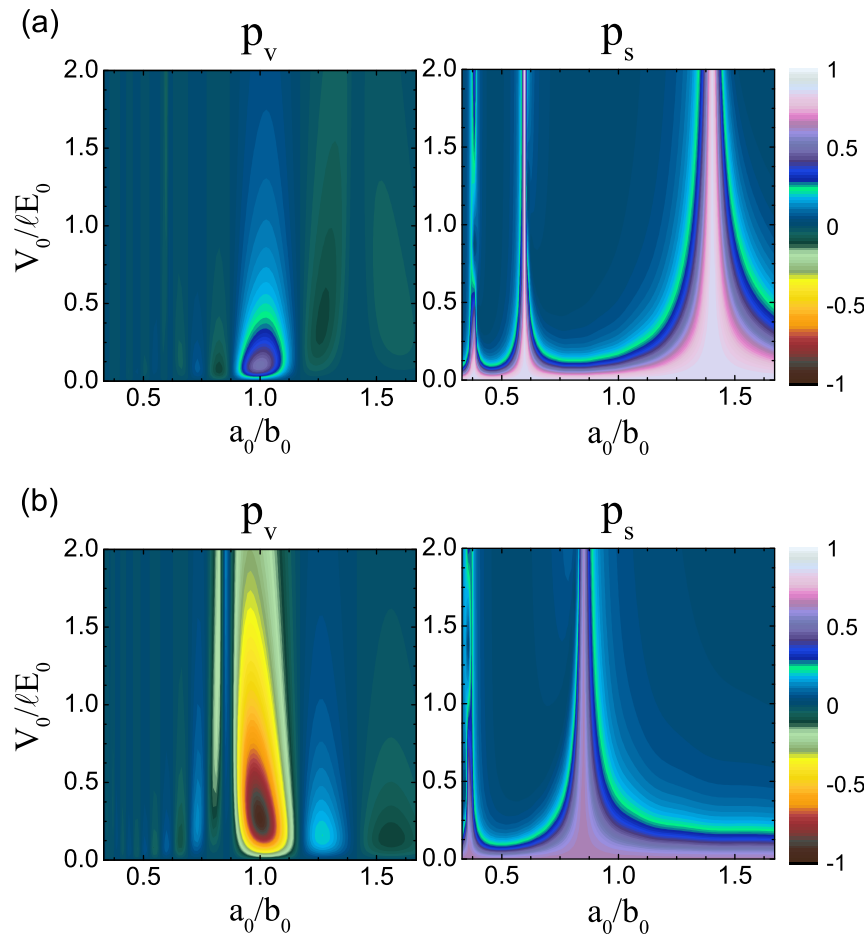

FIG. 9. (Color online) Contour plots of the valley $\left(p_{v}\right)$ and spin $\left(p_{s}\right)$ polarizations in terms of the strength $V_{0} / \ell E_{0}$ and period $a_{0} / b_{0}$ ratios for (a) $B=0.5 \mathrm{~T}$ and (b) $B=0.8 \mathrm{~T}$; $p_{v}$ is maximal for $a_{0}=b_{0}$; this holds also for other values of $B$.

\section{Hall conductivity}

We now treat the dc Hall conductivity $\sigma_{y x}^{\text {nd }}(0)$ in modulated silicene by setting $\omega=0$ in Eq. (18). To better assess the effect of the modulation we first consider the case where the applied electric field is uniform. To calculate $\sigma_{y x}$ we need the velocity operator $\hat{v}=\nabla_{\vec{\pi}} H$, which for the two valleys is given by $\hat{v}^{ \pm}=v_{F} \sigma_{x} \hat{e}_{x} \mp v_{F} \sigma_{y} \hat{e}_{y}$. Using the basis specified by Eqs. (5) and (7) we obtain

$$
\begin{aligned}
v_{x \zeta \zeta^{\prime}}^{+} v_{y \zeta^{\prime} \zeta}^{+} & =\left\langle n s_{z} p k_{x}\left|\hat{v}_{x}^{+}\right| n^{\prime} s_{z}^{\prime} p^{\prime} k_{x}^{\prime}\right\rangle\left\langle n^{\prime} s_{z}^{\prime} p^{\prime} k_{x}^{\prime}\left|\hat{v}_{y}^{+}\right| n s_{z} p k_{x}\right\rangle \\
& =i v_{F}^{2} \delta_{k_{x}, k_{x}^{\prime}} \delta_{s_{z}, s_{z}^{\prime}}\left[\left(\eta_{1}^{+\prime} \eta_{2}^{+}\right)^{2} \delta_{n^{\prime}, n-1}-\left(\eta_{1}^{+} \eta_{2}^{+\prime}\right)^{2} \delta_{n, n^{\prime}-1}\right]
\end{aligned}
$$

and

$$
\begin{aligned}
v_{x \zeta \zeta^{\prime}}^{-} v_{y \zeta^{\prime} \zeta}^{-} & =\left\langle n s_{z} p k_{x}\left|\hat{v}_{x}^{-}\right| n^{\prime} s_{z}^{\prime} p^{\prime} k_{x}^{\prime}\right\rangle\left\langle n^{\prime} s_{z}^{\prime} p^{\prime} k_{x}^{\prime}\left|\hat{v}_{y}^{-}\right| n s_{z} p k_{x}\right\rangle \\
& =i v_{F}^{2} \delta_{s_{z}, s_{z}^{\prime}} \delta_{k_{x}, k_{x}^{\prime}}\left[\left(\eta_{1}^{-} \eta_{2}^{-\prime}\right)^{2} \delta_{n^{\prime}, n-1}-\left(\eta_{1}^{-\prime} \eta_{2}^{-}\right)^{2} \delta_{n, n^{\prime}-1}\right] .
\end{aligned}
$$

For transitions to (or from) the zero LL we have

$$
\begin{aligned}
& \left\langle 0 s_{z} k_{x}\left|\hat{v}_{x}^{+}\right| n^{\prime} s_{z}^{\prime} p^{\prime} k_{x}^{\prime}\right\rangle\left\langle n^{\prime} s_{z}^{\prime} p^{\prime} k_{x}^{\prime}\left|\hat{v}_{y}^{+}\right| 0 s_{z} k_{x}\right\rangle \\
& =-i v_{F}^{2}\left(\eta_{2}^{+\prime}\right)^{2} \delta_{s_{z}, s_{z}^{\prime}} \delta_{k_{x}, k_{x}^{\prime}} \delta_{n^{\prime}, 1}, \\
& \left\langle n p s_{z} k_{x}\left|\hat{v}_{x}^{+}\right| 0 s_{z}^{\prime} k_{x}^{\prime}\right\rangle\left\langle 0 s_{z}^{\prime} k_{x}^{\prime}\left|\hat{v}_{y}^{+}\right| n s_{z} p k_{x}\right\rangle \\
& =i v_{F}^{2}\left(\eta_{2}^{+}\right)^{2} \delta_{s_{z}, s_{z}^{\prime}} \delta_{k_{x}, k_{x}^{\prime}} \delta_{n, 1}
\end{aligned}
$$

the results for the $K^{\prime}$ valley are given by Eqs. (25) and (26) with $\hat{v}_{x}^{+}$and $\eta_{2}^{+}$replaced by $\hat{v}_{x}^{-}$and $\eta_{1}^{-}$, respectively. 
Notice that all velocity elements are diagonal in the $k_{x}$ index (because of the presence of $\delta_{k_{x}, k_{x}^{\prime}}$ ) since the LLs are independent of $k_{x}$ for constant electric field. By substituting the above terms in Eq. (18) together with the unperturbed eigenvalues (4) one calculates the dc Hall conductivity.

Hall conductivity with field modulation. An inhomogeneous weak electric field perturbs the electronic wave functions and the cyclotron orbits. The perturbed states can be approximated by the first-order correction to the unperturbed wave functions as

$$
|\zeta\rangle=|\zeta\rangle^{0}+\sum_{\zeta^{\prime} \neq \zeta} \frac{\left(\ell E_{0}\right)_{\zeta^{\prime} \zeta}}{E_{\zeta}-E_{\zeta^{\prime}}}\left|\zeta^{\prime}\right\rangle^{0}
$$

where $|\zeta\rangle^{0} \equiv\left|n, s_{z}, p, k_{x}\right\rangle$ denotes the unperturbed state (5) or (7), and

$$
\begin{aligned}
& \left(\ell E_{0}\right)_{\zeta^{\prime} \zeta}={ }^{0}\left\langle\zeta^{\prime}\left|\ell E_{0} \sigma_{z}\right| \zeta\right\rangle^{0} \\
& =\ell E_{0} \delta_{s_{z}, s_{z}^{\prime}} \sqrt{n ! / n^{\prime} !} \\
& \times u^{\frac{n^{\prime}-n}{2}} e^{-u^{\prime} / 2} I_{\zeta^{\prime}, \zeta}^{ \pm}\left(u^{\prime}\right) \operatorname{Re}\left(i^{n^{\prime}-n} e^{i D x_{0}}\right),
\end{aligned}
$$

with

$$
\begin{aligned}
& I_{\zeta^{\prime}, \zeta}^{+}\left(u^{\prime}\right)=\eta_{1}^{+} \eta_{1}^{+\prime} L_{n}^{n^{\prime}-n}\left(u^{\prime}\right)-\eta_{2}^{+} \eta_{2}^{+1} \sqrt{\frac{n^{\prime}}{n}} L_{n-1}^{n^{\prime}-n}\left(u^{\prime}\right), \\
& I_{\zeta^{\prime}, \zeta}^{-}\left(u^{\prime}\right)=-\eta_{2}^{-} \eta_{2}^{-\prime} L_{n}^{n^{\prime}-n}\left(u^{\prime}\right)+\eta_{1}^{-} \eta_{1}^{-\prime} \sqrt{\frac{n^{\prime}}{n}} L_{n-1}^{n^{\prime}-n}\left(u^{\prime}\right) .
\end{aligned}
$$

Ignoring second-order perturbation terms we obtain analytically the off-diagonal velocity matrix elements. For instance,

$$
\begin{aligned}
\left\langle\zeta\left|\hat{v}_{x}^{+}\right| \zeta^{\prime}\right\rangle= & v_{F}^{0}\left\langle\zeta\left|\sigma_{x}\right| \zeta^{\prime}\right\rangle^{0} \\
& +\sum_{\zeta^{\prime \prime} \neq \zeta^{\prime}} \frac{v_{F}\left(\ell E_{0}\right)_{\zeta^{\prime \prime} \zeta^{\prime}}{ }^{0}}{E_{\zeta^{\prime}}-E_{\zeta^{\prime \prime}}}\left\langle\zeta\left|\sigma_{x}\right| \zeta^{\prime \prime}\right\rangle^{0} \\
& +\sum_{\zeta^{\prime \prime} \neq \zeta} \frac{v_{F}\left(\ell E_{0}\right)_{\zeta^{\prime \prime} \zeta}{ }^{0}}{E_{\zeta}-E_{\zeta}^{\prime \prime}}\left\langle\zeta^{\prime \prime}\left|\sigma_{x}\right| \zeta^{\prime}\right\rangle^{0},
\end{aligned}
$$

where

$$
{ }^{0}\left\langle\zeta\left|\sigma_{x}\right| \zeta^{\prime}\right\rangle^{0}=\eta_{1}^{+} \eta_{2}^{+\prime} \delta_{n, n^{\prime}-1}+\eta_{2}^{+} \eta_{1}^{+\prime} \delta_{n^{\prime}, n-1} .
$$

Similar expressions hold for $\hat{v}_{x}^{-}, \hat{v}_{y}^{+}, \hat{v}_{y}^{-}$, etc.

Figure 10(a) shows the Hall conductivity $\sigma_{y x}$ versus the magnetic field $B$ in the presence of a uniform electric field and of a modulated one. One clearly sees the well-known plateaus which result from the Landau quantization of the cyclotron orbits. The transition between the plateaus occur precisely at the magnetic fields at which the Fermi level $E_{F}$ and the corresponding Landau index $n_{F}$ change sharply as indicated by the arrows. (This results in the Shubnikov-de Haas oscillations.) When a fixed nonzero field $E_{z}$ is applied, all LLs split due to the spin and valley degrees of freedom and, as a result, the number of sharp fluctuations in $E_{F}$ increases. This leads to new extra plateaus, within the same integer $n$ LL plateaus. Since the valley splitting is very weak for fixed $E_{z}$, the extra plateaus are mostly due to spin resolution. In contrast, by applying a modulated $E_{z}$ field, the LL splitting due to the valley degree of freedom becomes wider and comparable to the spin splitting, see Fig. 3(a). Therefore, the large plateaus related to fixed fields are replaced
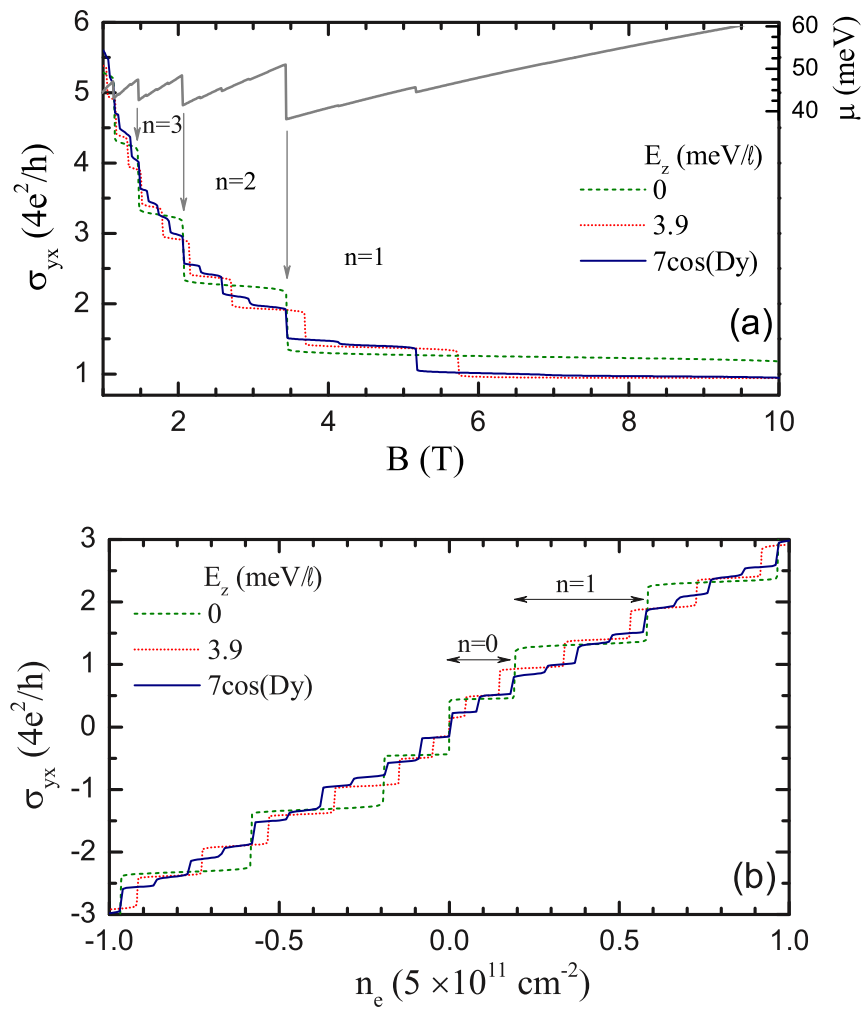

FIG. 10. (Color online) (a) Hall conductivity $\sigma_{y x}$ as a function of the magnetic field $B$ at $T=2 \mathrm{~K}$ and $n_{e}=5 \times 10^{11} \mathrm{~cm}^{-2}$. The dashed and dotted curves are for the uniform fields $E_{z}=0$ and $E_{z}=3.9 \mathrm{meV} / \ell$, respectively; the solid curve is for a modulated field with $E_{0}=7 \cos (D y) \mathrm{meV} / \ell$. The upmost (gray) curve shows the chemical potential $\mu$ versus the field $B$ which corresponds approximately to the $E_{F}$ variations since $T$ is very low. A constant electric field doubles the number of plateaus and the modulated field $E_{z}$ doubles it again. (b) Hall conductivity versus the electron density for $B=2 \mathrm{~T}, T=2 \mathrm{~K}$ and the same field values as in (a).

by a series of steps and small plateaus due to the intra-LL spin or valley transitions. Since the occupation capacity of each LL depends only on the magnetic field, through the flux density $1 / 2 \pi l_{B}^{2}$, a transition between plateaus occurs when $n_{e} /\left(1 / 2 \pi l_{B}^{2}\right)$ becomes an integer. Ideally this occurs at the fields $B=n_{e} \phi_{0} / m$ for $T=0$ and the LL broadening vanishingly small. For this ideal case the location of plateaus transitions is independent of the modulation features such as period, amplitude, etc. A change in the modulation features affects only the height of the steps between the new plateaus.

Figure 10(b) shows the Hall conductivity $\sigma_{y x}$ versus the electron density $n_{e}$ for a magnetic field $B=2 \mathrm{~T}$. By applying a uniform nonzero electric field the number of plateaus doubles and interestingly, with a modulated field $E_{z}$ a series of steps and small plateaus are created. As discussed, these plateaus result from the sharp changes in $E_{F}$ and from the intra-LL transitions when the LL splitting is strong. The distance between the plateaus is $\delta n_{e}=1 / 2 \pi l_{B}^{2} \approx 0.1 n_{0}$, with $n_{0}=5 \times 10^{11} \mathrm{~cm}^{-2}$ (see the width of the steps shown by the solid curve). Note that the new plateaus can be observed in the experiment as long as the broadening of LLs due to the scattering mechanisms, e.g., 
by impurities or phonons, is much smaller than the spin and valley splitting induced by the modulated field $E_{z}$.

\section{Magnetoresistivity}

We now turn to the evaluation of the magnetoresistivity in modulated silicene by calculating its longitudinal and transverse components. To this end we need to evaluate the collisional contribution to the conductivity along and perpendicular to the direction of the modulation. For a 2D electron system, the magnetoresistivity tensor, with $S=\sigma_{x x} \sigma_{y y}-\sigma_{x y} \sigma_{y x}$, takes the form

$$
\rho=\left[\begin{array}{ll}
\rho_{x x} & \rho_{x y} \\
\rho_{y x} & \rho_{y y}
\end{array}\right]=\frac{1}{S}\left[\begin{array}{cc}
\sigma_{y y} & -\sigma_{x y} \\
-\sigma_{y x} & \sigma_{x x}
\end{array}\right] .
$$

The collisional conductivity is given by Eq. (17). Ignoring scattering by phonons at very low temperatures, $\sigma_{\mu \mu}^{\mathrm{col}}$ is mainly determined from scattering by impurities. We assume that the impurities are randomly distributed and their density is $n_{i}$. Then the transition rate $W_{\zeta, \zeta^{\prime}}$ is

$$
W_{\zeta, \zeta^{\prime}}=\frac{2 \pi n_{i}}{\hbar S_{0}} \sum_{\mathbf{q}}\left|U_{q}\right|^{2}\left|\left\langle\zeta\left|e^{i \mathbf{q} \cdot \mathbf{r}}\right| \zeta^{\prime}\right\rangle\right|^{2} \delta\left(E_{\zeta}-E_{\zeta^{\prime}}\right),
$$

where $\mathbf{q}=\mathbf{k}-\mathbf{k}^{\prime}$ is the scattered wave vector, $U_{q}$ is the Fourier transform of the impurity potential, and $\mathbf{r}$ is the electron's position vector. We take the screened impurity potential as $U(\mathbf{r})=U_{0} e^{-q_{s} r} / r$, where $U_{0}=e^{2} / 4 \pi \epsilon_{r} \epsilon_{0}, \epsilon_{r}$ is the dielectric constant, $\epsilon_{0}$ is the vacuum permittivity, and $q_{s}$ is the screening wave vector which characterizes the potential range of the scatterers. The Fourier transform of $U(\mathbf{r})$ is $U_{q}=2 \pi U_{0}\left(q^{2}+q_{s}^{2}\right)^{-1 / 2}$, which for short-range scattering $q \ll q_{s}$, is approximately equal to $2 \pi U_{0} / q_{s}$.

The matrix elements in Eq. (33) can be calculated using standard formulas. For the $K$ valley, with $\Theta=l_{B}^{2} q_{y}\left(k_{x}-\right.$ $\left.q_{x} / 2\right)$ and $\gamma=l_{B}^{2} q^{2} / 2$, we obtain

$$
\begin{aligned}
{ }^{0}\left\langle\zeta\left|e^{i \mathbf{q} \cdot \mathbf{r}}\right| \zeta^{\prime}\right\rangle^{0}= & \delta_{k_{x}, k_{x}^{\prime}+q_{x}} \delta_{s_{z}, s_{z}^{\prime}} e^{i \Theta-\gamma / 2} \\
& \times\left(\eta_{1}^{+} \eta_{1}^{+\prime} F_{n, n^{\prime}}+\eta_{2}^{+} \eta_{2}^{+\prime} F_{n-1, n^{\prime}-1}\right),
\end{aligned}
$$

and for the $K^{\prime}$ one

$$
\begin{aligned}
{ }^{0}\left\langle\zeta\left|e^{i \mathbf{q} \cdot \mathbf{r}}\right| \zeta^{\prime}\right\rangle^{0}= & \delta_{k_{x}, k_{x}^{\prime}+q_{x}} \delta_{s_{z}, s_{z}^{\prime}} e^{i \Theta-\gamma / 2} \\
& \times\left(\eta_{2}^{-} \eta_{2}^{-\prime} F_{n, n^{\prime}}+\eta_{1}^{-} \eta_{1}^{-\prime} F_{n-1, n^{\prime}-1}\right)
\end{aligned}
$$

where

$$
F_{n, n^{\prime}}= \begin{cases}\sqrt{\frac{2^{n} n^{\prime} !}{2^{n^{\prime}} n !}}\left(-v^{*}\right)^{n-n^{\prime}} L_{n}^{n-n^{\prime}}(\gamma), & n^{\prime} \leqslant n, \\ \sqrt{\frac{2^{n^{\prime} n !}}{2^{n} n^{\prime} !}} v^{n^{\prime}-n} L_{n^{\prime}}^{n^{\prime}-n}(\gamma), & n \leqslant n^{\prime},\end{cases}
$$

with $v=l_{B}\left(i q_{y}+q_{x}\right) / 2$. Note that in calculating the transition rate $W_{\zeta \zeta^{\prime}}$, the major contribution results from the unperturbed state $|\zeta\rangle^{0}=\left|n, s_{z}, p, k_{x}\right\rangle^{0}$, that is, $\left\langle\zeta\left|e^{i \mathbf{q} \cdot \mathbf{r}}\right| \zeta^{\prime}\right\rangle \approx{ }^{0}\left\langle\zeta\left|e^{i \mathbf{q} \cdot \mathbf{r}}\right| \zeta^{\prime}\right\rangle^{0}$. Therefore, by defining $n_{<} \equiv \min \left(n, n^{\prime}\right)$ and $n_{>} \equiv \max \left(n, n^{\prime}\right)$ we find

$$
\left.W_{\zeta \zeta^{\prime}}^{0} \approx \frac{2 \pi n_{i}}{\hbar S_{0}} \sum_{\mathbf{q}}\left|U_{q}\right|^{2} \delta_{k_{x}, k_{x}^{\prime}+q_{x}} \delta_{s_{z}, s_{z}^{\prime}} J_{n n^{\prime}, p p^{\prime}}^{ \pm}\left(s_{z}\right)\right|^{2} \delta\left(E_{\zeta}-E_{\zeta^{\prime}}\right),
$$

where

$$
\begin{aligned}
\left|J_{n n^{\prime}, p p^{\prime}}^{+}\left(s_{z}\right)\right|^{2}= & \frac{n_{>} !}{n_{<} !} \gamma^{n_{>}-n_{<}} e^{-\gamma}\left[\eta_{1}^{+} \eta_{1}^{+\prime} L_{n_{<}}^{n_{>}-n_{<}}(\gamma)\right. \\
& \left.+\eta_{2}^{+} \eta_{2}^{+\prime} \sqrt{\frac{n_{<}}{n_{>}}} L_{n_{<}-1}^{n_{>}-n_{<}}(\gamma)\right]^{2}
\end{aligned}
$$

and

$$
\begin{aligned}
\left|J_{n n^{\prime}, p p^{\prime}}^{-}\left(s_{z}\right)\right|^{2}= & \frac{n_{>} !}{n_{<} !} \gamma^{n_{>}-n_{<}} e^{-\gamma}\left[\eta_{2}^{-} \eta_{2}^{-\prime} L_{n_{<}}^{n_{>}-n_{<}}(\gamma)\right. \\
& \left.+\eta_{1}^{-} \eta_{1}^{-\prime} \sqrt{\frac{n_{<}}{n_{>}}} L_{n_{<}-1}^{n_{>}-n_{<}}(\gamma)\right]^{2} .
\end{aligned}
$$

Using Eqs. (37) and $y_{\zeta}-y_{\zeta^{\prime}}=l_{B}^{2}\left(k_{x}-k_{x}^{\prime}\right)=l_{B}^{2} q_{x}$, the collisional conductivity Eq. (17) takes the form

$$
\begin{aligned}
\sigma_{y y}^{\mathrm{col}}= & \frac{e^{2}}{h} \frac{2 \beta \pi^{2} n_{i} l_{B}^{4}}{S_{0}^{2}} \sum_{n, n^{\prime}} \sum_{p, p^{\prime} k_{x}, s_{z}, \pm} \sum_{\mathbf{q}}\left|U_{q}\right|^{2} q_{x}^{2} f\left(E_{n, s_{z}, p}^{ \pm}\right) \\
& \times\left[1-f\left(E_{n^{\prime}, s_{z}, p^{\prime}}^{ \pm}\right)\right]\left|J_{n n^{\prime}, p p^{\prime}}^{ \pm}\left(s_{z}\right)\right|^{2} \\
& \times \delta\left(E_{n, s_{z}, p}^{ \pm}-E_{n^{\prime}, s_{z}, p^{\prime}}^{ \pm}+\Delta_{n n^{\prime}}\right),
\end{aligned}
$$

where $E_{n, s_{z}, p}^{ \pm}$is the unperturbed eigenvalue (4) and $\Delta_{n n^{\prime}}$ is the difference between the bandwidths of the $n$ and $n^{\prime}$ LLs, cf. Eqs. (9)-(14). At sufficiently low temperatures the dominant scattering is elastic and the transition between the electron-hole states is usually suppressed (this is further supported at low magnetic fields by the fact that the SOI gaps out the electron-hole states even for $n=0$ ). Therefore, we can approximate the $\delta$ function by a Lorentzian broadening of zero shift and of width $\Gamma_{n}$ which peaks around $n=n^{\prime}$. Now using polar coordinates we make the changes $q_{x} \rightarrow q \cos \varphi$ and $\sum_{\mathbf{q}} \rightarrow\left(S_{0} / 4 \pi^{2} l_{B}^{2}\right) \int_{0}^{2 \pi} d \varphi \int_{0}^{\infty} d \gamma, \gamma=q^{2} / 2 \ell_{B}^{2}$. Eventually, substituting $\left|J_{n n, p p}^{ \pm}\left(s_{z}\right)\right|^{2}=e^{-\gamma}\left|G_{n, s_{z}, p}^{ \pm}(\gamma)\right|^{2}$, using $\sum_{k_{x}} \rightarrow$ $L_{x} \int_{0}^{k_{0}} d k_{x} / 2 \pi$, and setting $A=2 \pi n_{i} U_{0}^{2} / \hbar^{2} v_{F}^{2} q_{s}^{2}$, Eq. (40) becomes

$$
\sigma_{y y}^{\mathrm{col}, 0}=\frac{e^{2}}{h} A \sum_{n, s_{z}, p} P_{n, s_{z}, p} \int_{0}^{\infty} \gamma e^{-\gamma}\left|G_{n, s_{z}, p}^{ \pm}(\gamma)\right|^{2} d \gamma,
$$

with

$$
P_{n, s_{z}, p}=\left(\hbar^{2} v_{F}^{2} / L_{y} \Gamma_{n}\right) \int_{0}^{k_{0}}[\partial f(E) / \partial E]_{E=E_{n, s_{z}, p}^{ \pm}} d k_{x} .
$$

For a Thomas-Fermi screening wave vector as large as $q_{s} \approx 5 \times 10^{8} \mathrm{~m}^{-1}$, an impurity density $n_{i}=1 \times 10^{9} \mathrm{~cm}^{-2}$, and $\epsilon_{r} \approx 4$ we attain $A \approx 2.5 \times 10^{-4}$. Note that the Thomas-Fermi wave vector of a screened charge in a 2D Dirac gas is about $e^{2} \sqrt{n_{e}} / \sqrt{\pi} \epsilon_{r} \epsilon_{0} \hbar v_{F}$ (in SI units) [24]. The integral over $\gamma$ in Eq. (41), denoted as $I^{ \pm}$, can be calculated analytically using the orthogonality of the Laguerre polynomials. The result is

$$
I^{+}=\left|\eta_{1}^{+}\right|^{4}(2 n+1)+\left|\eta_{2}^{+}\right|^{4}(2 n-1)-2 n\left|\eta_{1}^{+}\right|^{2}\left|\eta_{2}^{+}\right|^{2},
$$

for the $K$ valley. Similarly for the $K^{\prime}$ valley the result is

$$
I^{-}=\left|\eta_{2}^{-}\right|^{4}(2 n+1)+\left|\eta_{1}^{-}\right|^{4}(2 n-1)-2 n\left|\eta_{1}^{-}\right|^{2}\left|\eta_{2}^{-}\right|^{2} \text {. }
$$

The potential due to scattering centers, such as impurities, also broadens the LLs. Similarly, the induced LL width differs for every LL depending on the Landau index $n$. Here, 


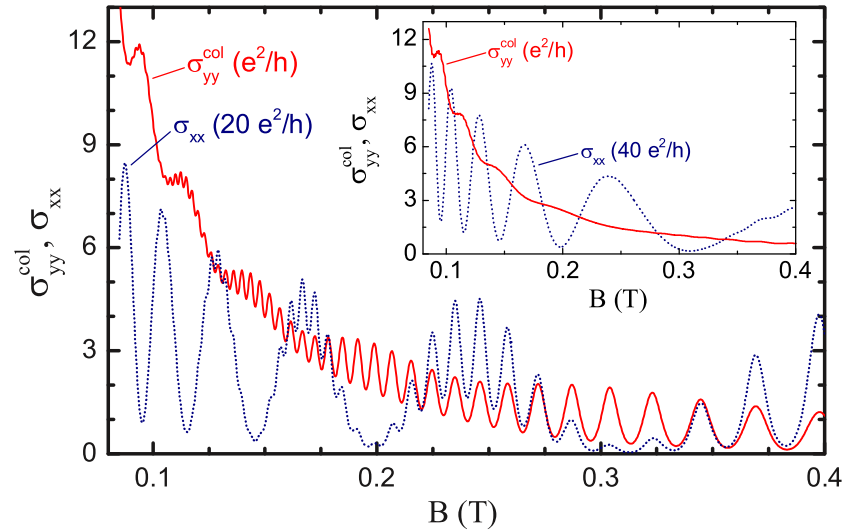

FIG. 11. (Color online) (a) The longitudinal conductivities $\sigma_{y y}^{\text {col }}$ and $\sigma_{x x}$ as function of the field $B$ for $T=2 \mathrm{~K}, A=2.5 \times 10^{-4}$, $E_{0}=1 \mathrm{meV}, V_{0}=0.5 \mathrm{meV}$, and $a_{0}=b_{0}=300 \mathrm{~nm}$. The inset shows the same $\sigma_{\mu \mu}$ components for $T=6 \mathrm{~K}$. For low fields the collisional and diffusive conductivities oscillate with a phase difference of $180^{\circ}$ but upon increasing $B$ this phase difference gradually decreases and at higher $B$ they oscillate in phase.

for simplicity, we omit this width dependence on $n$ and $k_{x}$ as it is smaller than that for a weak modulation. The collisional contribution to the longitudinal conductivity is shown in Fig. 11 as a function of the magnetic field $B$ for two low temperatures $T=2$ and $6 \mathrm{~K}$. Since the LLs increase proportional to $\sqrt{n}$ for $\bar{\lambda}_{\text {so }} \ll n$, the Lorentzian width $\Gamma_{n}$ varies with the Landau index approximately as $\Gamma_{n} \propto \hbar \omega_{c} / \sqrt{n}$. Also, in the calculation of the DOS we assume a slightly larger broadening in Eq. (15), $\Gamma=0.1 \hbar \omega_{c}$, because of the LL broadening due to the impurities. As seen, for increasing $B$ the conductivity decreases, whereas the amplitude of the tiny oscillations increases, notice the different scales of its two components. In a strong magnetic field the diffusive motion of electrons is suppressed because the cyclotron orbits become smaller and this reduces the conductivity. Notice also that the decrease in oscillation amplitude is faster in the collisional than in the diffusive conductivity which is also the case in a 2DEG [16] and in graphene [18,19].

We plot the magnetoresistivity tensor components in Fig. 12 for $\sigma_{0}=10^{3} e^{2} / h$, using Eq. (32) with the total longitudinal

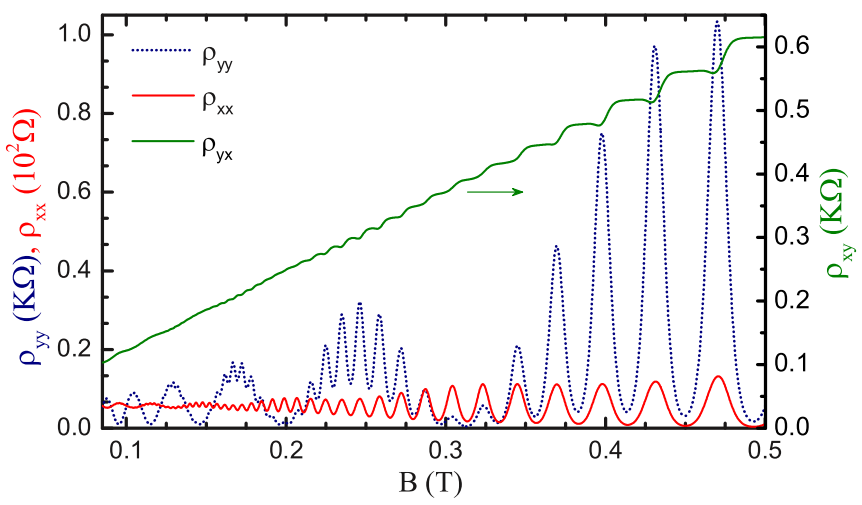

FIG. 12. (Color online) (a) The longitudinal magnetoresistivity components as function of the magnetic filed $B$ for $T=2 \mathrm{~K}$, $E_{0}=1 \mathrm{meV}, V_{0}=0.5 \mathrm{meV}$, and $a_{0}=b_{0}=300 \mathrm{~nm}$. The curve referred to the right axis shows the Hall magnetoresistivity $\rho_{x y}$.

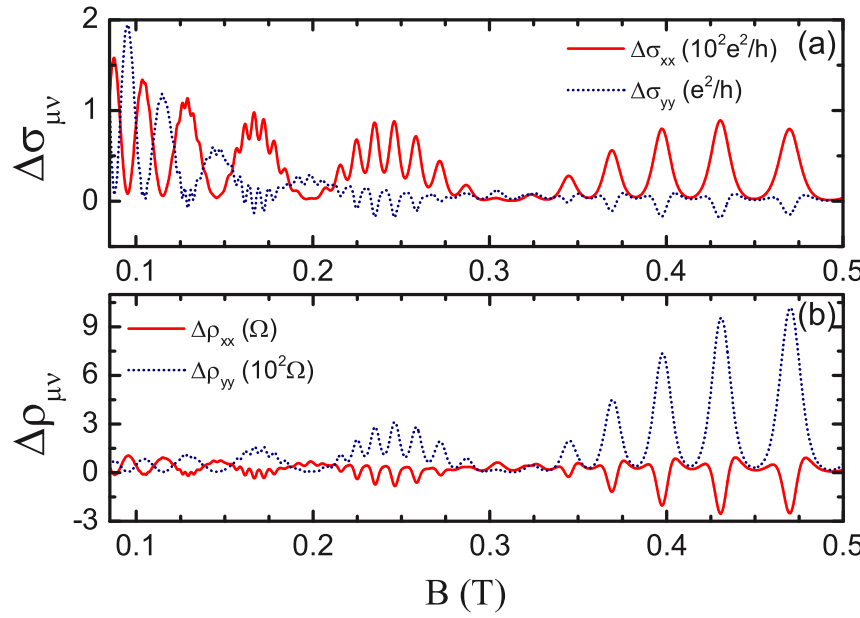

FIG. 13. (Color online) (a) The correction to the longitudinal conductivity due to the modulation. (b) The same as in (a) but for the magnetoresistivity components.

conductivity given by $\sigma_{x x}=\sigma_{x x}^{\text {dif }}+\sigma_{x x}^{\text {col }}$ and $\sigma_{y y}=\sigma_{y y}^{\text {col }}$. In contrast to the magnetoconductivity, increasing $B$ leads to an increase in the oscillation amplitude. These commensurability oscillations in $\rho_{\mu \mu}$ originate from the LL broadening due to a weak modulation, whereas the Shubnikov-de Haas oscillations, which occur when the Fermi level passes through successive LLs, start at higher $B$. As in a 2DEG, the transitions between plateaus in the magnetoresistivity component $\rho_{x y}$, shown on the right axis, coincide with the maxima in $\rho_{x x}$. At very low $B$ though, $\rho_{x y}$ decreases almost linearly with $B$ and the plateaus disappear.

Finally, for a closer comparison with the usual 2DEG, in Fig. 13 we show the corrections due to the modulation $\Delta \sigma_{y y}$, $\Delta \sigma_{x x}, \Delta \rho_{y y}$, and $\Delta \rho_{x x}$. As in a $2 \mathrm{DEG}$, the commensurability oscillations in $\Delta \sigma_{y y}$ and $\Delta \sigma_{x x}$ shown in Fig. 13(a), as well as in $\Delta \rho_{y y}$ and $\Delta \rho_{x x}$ shown in Fig. 13(b), differ in phase by $180^{\circ}$ for $B \leqslant 0.3 \mathrm{~T}$, whereas for higher $B$ the oscillation pattern, though similar, is more complex.

\section{CONCLUSIONS}

We studied magnetotransport properties of low-energy fermions in silicene in the presence of a weak field and/or potential periodic modulations and of a weak magnetic field normal to the silicene plane. Due to the strong spin-orbit interaction all LLs split. Without electric field the split LLs are valley degenerate. Although the application of a constant electric field lifts this degeneracy, the valley splitting is still weak.

The weak field and potential modulations broaden the LLs into bands and lead to a diffusive conductivity in the modulation direction. At very weak magnetic fields, a weak potential modulation can induce a beating pattern in the commensurability oscillations, depending on the strength of constant $E_{z}$, but this is not the case when only $E_{z}$ is modulated. We demonstrated that the spin and valley splitting due to only a potential modulation is very weak. In contrast, a spatially modulated electric field leads to largely spin-resolved contributions to the diffusive conductivity. However, the amplitude of the 
corresponding spin-resolved Weiss oscillations is small. To resolve this, we demonstrated that a combination of field and potential modulations can result in spin- and valley-resolved Weiss oscillations and restore the oscillation amplitude. We also quantified the valley and spin polarizations versus the field $B$ in Fig. 8 and as functions of the ratios of the modulation strengths and periods, cf. Fig. 9. One can clearly observe the same spin gaps in the Weiss oscillations of the diffusive conductivity as in Fig. 8. This does not occur in graphene because of its very weak spin-orbit interaction. Furthermore, by calculating the collisional contribution, we highlighted the similarities in and the differences from the commensurability oscillations in the conductivities/resistivities and the corresponding ones in graphene and in a 2DEG.

We also studied the Hall conductivity $\sigma_{y x}$ and showed that the field modulation creates extra narrow plateaus within the standard ones or integer $n$ LL plateaus. All plateaus are due to sharp changes of the Fermi level, as it moves through the LLs, and the new ones result from the lifting of the spin and valley degeneracies and the corresponding transitions between the four $(n \geqslant 1)$ or two $(n=1)$ sublevels. In fact, the step structure, within the same integer $n$ LL plateaus, replaces the latter by an inclined series of narrow plateaus and steps. That is, the standard integer $n$ LL plateaus are destroyed.

\section{ACKNOWLEDGMENTS}

Our work was supported by the Flemish Science Foundation (FWO-VI), the Methusalem Foundation of the Flemish Government, and by the Canadian NSERC Grant No. OGP0121756.
[1] Z. Ni, Q. Liu, K. Tang, J. Zheng, J. Zhou, R. Qin, Z. Gao, D. Yu, and J. Lu, Nano Lett. 12, 113 (2011).

[2] P. Vogt, P. De Padova, C. Quaresima, J. Avila, E. Frantzeskakis, M. C. Asensio, A. Resta, B. Ealet, and G. Le Lay, Phys. Rev. Lett. 108, 155501 (2012); A. Fleurence, R. Friedlein, T. Ozaki, H. Kawai, Y. Wang, and Y. Yamada-Takamura, ibid. 108, 245501 (2012).

[3] G. G. Guzmán-Verri and L. C. Lew Yan Voon, Phys. Rev. B 76, 075131 (2007); S. Lebègue and O. Eriksson, ibid. 79, 115409 (2009).

[4] Y. Cai, C.-P. Chuu, C. M. Wei, and M. Y. Chou, Phys. Rev. B 88, 245408 (2013).

[5] H. Liu, J. Gao, and J. Zhao, J. Phys. Chem. C 117, 10353 (2013).

[6] M. Neek-Amal, A. Sadeghi, G. R. Berdiyorov, and F. M. Peeters, Appl. Phys. Lett. 103, 261904 (2013).

[7] D. Chiappe, E. Scalise, E. Cinquanta, C. Grazianetti, B. V. Broek, M. Fanciulli, M. Houssa, and A. Molle, Adv. Mater. 26, 2096 (2014).

[8] C.-C. Liu, H. Jiang, and Y. Yao, Phys. Rev. B 84, 195430 (2011); C.-C. Liu, W. Feng, and Y. Yao, Phys. Rev. Lett. 107, 076802 (2011).

[9] M. Ezawa, Phys. Rev. Lett. 109, 055502 (2012); New J. Phys. 14, 033003 (2012).

[10] C. J. Tabert and E. J. Nicol, Phys. Rev. Lett 110, 197402 (2013).
[11] H. Pan, Z. Li, C.-C. Liu, G. Zhu, Z. Qiao, and Y. Yao, Phys. Rev. Lett. 112, 106802 (2014).

[12] M. Tahir and U. Schwingenschlögl, Appl. Phys. Lett. 101, 132412 (2012).

[13] D. Xiao, G.-B. Liu, W. Feng, X. Xu, and W. Yao, Phys. Rev. Lett 108, 196802 (2012).

[14] SK Firoz Islam and Tarun Kanti Ghosh, J. Phys.: Condens. Matter 26, 335303 (2014).

[15] D. Weiss, K. V. Klitzing, K. Ploog, and G. Weimann, Europhys. Lett. 8, 179 (1989).

[16] F. M. Peeters and P. Vasilopoulos, Phys. Rev. B. 46, 4667 (1992).

[17] X. F. Wang, P. Vasilopoulos, and F. M. Peeters, Phys. Rev. B 71, 125301 (2005).

[18] A. Matulis and F. M. Peeters, Phys. Rev. B 75, 125429 (2007).

[19] R. Nasir, K. Sabeeh, and M. Tahir, Phys. Rev. B 81, 085402 (2010).

[20] M. Zarenia, P. Vasilopoulos, and F. M. Peeters, Phys. Rev. B 85 , 245426 (2012).

[21] M. Charbonneau, K. M. Van Vliet, and P. Vasilopoulos, J. Math. Phys. 23, 318 (1982).

[22] P. Vasilopoulos, Phys. Rev. B 32, 771 (1985).

[23] M. O. Goerbig, Rev. Mod. Phys. 83, 1193 (2011).

[24] T. Stauber, N. M. R. Peres, and F. Guinea, Phys. Rev. B 76, 205423 (2007). 